\title{
En torno a la identidad romana: balance crítico de sus teorías contemporáneas*
}

\author{
About Roman Identity: Critical Balance of its Contemporary Theories
}

Daniel Nieto Orriols ${ }^{* *}$

\section{RESUMEN}

El presente artículo ofrece un análisis crítico de las teorías contemporáneas que abordan la identidad romana de la Antigüedad Clásica. Identificamos un traspaso entre perspectivas homogeneizantes hacia otras que advierten la relevancia de grupos más pequeños como agentes de su propia identidad. Desde su concepción de la identidad y sus factores de desarrollo, en la actualidad identificamos tres líneas teóricas generales, de las que se han derivado tres propuestas específicas. En primer lugar, las que advierten la identidad en su relación con la romanización. En segundo lugar, las que rechazan dicho concepto y relevan el de etnicidad, y, a partir de este, el de rechazo y discrepancia. En tercer lugar, y como vinculación entre ambas, la identidad cultural. Derivado de esta última encontramos, finalmente, una importante vía cuyos trabajos comparecen al referir la identidad romana como civitas.

Palabras clave: identidad romana, romanización, etnicidad, identidad cultural, civitas

\section{ABSTRACT}

This article offers a critical analysis of contemporary theories addressing the Roman identity of Classical Antiquity. We identified a transfer among the homogenizing perspectives to others that make visible the relevance of smaller groups as agents of their own identity. From this conception of identity and its development factors, we recognized three general theories, from which three specific proposals have been derived: Firstly, that recognizing the identity in its relation with the romanization; secondly, that rejecting this concept and revealing that of ethnicity, and, from this, that of rejection and discrepancy; and finally, that linking previous theories - cultural identity. From this last theory, we found a relevant way whose works seems to refer to the Roman identity as civitas.

Keywords: Roman identity, romanization, ethnicity, cultural identity, civitas.

Recibido: junio 2019

Aceptado: diciembre 2019

\footnotetext{
* Este trabajo es resultado de mi tesis doctoral: Salustio y la identidad. Una aproximación al ser romano en época de crisis. Pontificia Universidad Católica de Valparaíso, 2018.

** Doctor en Historia y Magíster en Historia, Pontificia Universidad Católica de Valparaíso. Académico regular y Profesor de Historia Antigua, Universidad Andrés Bello. Email: daniel.nieto@unab.cl
} 


\section{Introducción}

En el presente trabajo ofrecemos un recorrido crítico por las principales corrientes contemporáneas que estudian la identidad romana. Aludimos los principales problemas y conceptos que comparecen en el análisis de la romanidad ${ }^{1}$, desde fines del siglo XIX hasta nuestros tiempos, a partir tres perspectivas centrales en la discusión historiográfica: romanización, etnicidad e identidad cultural. Nos proponemos, por un lado, entregar un estado del arte actualizado sobre la identidad romana y los problemas conceptuales y contextuales que le revisten; $y$, por otro, evidenciar cómo los paradigmas culturales, desde la teoría del discurso y en un análisis a diversas escalas, han posibilitado el diálogo entre corrientes contrapuestas y han generado la relectura de perspectivas que, a pesar de la profunda crítica en su contra, en su versión actualizada parecen responder de forma clara al problema de la identidad en Roma.

El análisis de la identidad romana constituye un tema de reciente investigación historiográfica. Solo en la segunda midad del siglo XX la identidad se estableció como objeto de investigación en los estudios del mundo antiguo. Si bien el tratamiento de la cultura y de las características sociopolíticas de Roma se venía realizando en la historiografía del siglo XIX, esta no atendía la romanidad en sí misma, pues los fundamentos del rankeanismo, de profunda influencia en los estudios de la urbs, centraron su atención en las instituciones jurídicas que caracterizaban a Roma y a aquellos que vivían en su comunidad política ${ }^{2}$. Se trata de una mirada que, sustentada en la concepción moderna de la nación ${ }^{3}$ y en el contexto del imperialismo europeo ${ }^{4}$, desarrolló una interpretación también moderna de la antigüedad ${ }^{5}$, cuya propuesta sobre el ser romano alberga uno de los principales conceptos del debate historiográfico contemporáneo: la romanización.

Tanto el proceso de la descolonización como el surgimiento de los paradigmas de postguerra generaron un ingiente debate sobre la teoría de la romanización, y, a partir de la década de 1970, los estudios de la identidad romana han migrado desde las visiones homogeneizantes,

\footnotetext{
${ }^{1}$ Utilizamos los conceptos romanidad, romanitas y ser romano como identidad romana. Sobre algunos detalles del concepto y su relación con la identidad véase Bancalari, Alejandro. 2007. Orbe romano e imperio global. La romanización desde Augusto a Caracalla, Santiago, Editorial Universitaria, pp. 56-57.

${ }^{2}$ Hurtado, Miguel. 2005. Introducción a la historia antigua. Caracas, Fondo de Humanidades, pp. 40-41.

${ }^{3}$ Al respecto véase Hobsbawm, Eric. 2004. Naciones y nacionalismo desde 1789, Barcelona, Crítica, p. 23.

${ }^{4}$ Véase Wulff, Fernando. 2007. "Las provincias de Hispania en época Republicana: una reflexión sobre enfoques y perspectivas", en Santos, Juan y Torregaray, Elena (eds.). Laudes provinciarum: retórica y política en la representación del imperio romano, Vitoria, Universidad del País Vasco, p. 4.

${ }^{5}$ Véase Buono-Core, Raúl. 2006. “Un breve análisis de la historiografía griega sobre la antigua Grecia en los siglos XIX y XX", en Europa, №4, pp. 55-68; Sancho, Laura (coord.). 2015. La Antigüedad como paradigma. Espejismos, mitos y silencios en el uso de la historia del mundo clásico por los modernos. Zaragoza, Universidad de Zaragoza, pp. 3 y ss.
} 
sustentadas en el rol del Estado como agente identitario, hacia otras menos ambiciosas, centradas en pequeños grupos sociales agentes de su propia identidad. Perspectivas que, aunque opuestas, convergen en el tratamiento de un concepto cuyo análisis ha suscitado una profunda reflexión de las características de la identidad romana en términos compositivos y adquisitivos. Compositivos, en la medida que advierte lo que significó -o no- ser romano; y adquisitivos, en tanto alude las modalidades en que la romanitas fue adquirida, promovida, impuesta o rechazada por las comunidades en contacto con Roma.

Como explicación de la incorporación de pueblos a la cultura romana, en su planteamiento original la romanización es un concepto ambivalente y criticado, y, por sus referencias a la identidad, se ha establecido como eje de los principales estudios. Estos análisis han generado un debate entre visiones universalistas, relacionadas con la romanización-imperialismo, y otras de carácter más bien local, que, identificando nuevos factores de la identidad, han posibilitado distinguir características particularizadas. A partir del modo en que conciben la identidad y sus factores de desarrollo, en la actualidad identificamos tres líneas teóricas generales, de las que se han derivado, a su vez, tres propuestas específicas. En primer lugar, las visiones históricas, que advierten la identidad en su relación con la romanización, tanto en su versión tradicional como en otra renovada. En segundo lugar, vinculadas a la arqueología, las que rechazan dicho concepto, relevan el de etnicidad y, a partir de este, el de rechazo y discrepancia. En tercer lugar, y como vinculación entre las visiones histórica y arqueológica, la identidad cultural, que promueve una perspectiva más amplia en cuanto a fuentes y vías de análisis. Derivado de esta última encontramos una importante vía cuyos trabajos comparecen al referir la identidad romana como civitas.

\section{Identidad y romanización: de la visión tradicional a la visión actual}

La conceptualización de la romanización a fines del siglo XIX e inicios del XX ha conformado uno de los enfoques más relevantes en los estudios de la identidad. Es necesario distinguir, no obstante, dos posturas o momentos de esta teoría. En primer lugar, arraigada en la concepción moderna, una visión que concibe la romanización como proceso unívoco, transversal y dirigido desde la autoridad política romana hacia las comunidades foráneas; $y$, en segundo lugar, a propósito de las críticas postcoloniales y del surgimiento de paradigmas culturales, una mirada heterogénea que considera nuevas formas de traspaso cultural y diversos agentes en la conformación identitaria en las provincias.

La primera versión de la romanización -que, siguiendo a Bancalari ${ }^{6}$, denominaremos clásicaresultó de una interpretación generada en la época de los imperialismos, fundada en sus paradigmas historiográficos y en sus intereses político-ideológicos. Su propósito fue explicar las

\footnotetext{
${ }^{6}$ Bancalari, Alejandro. 2006. Orbe romano, p. 68.
} 
características y los efectos sociales de la expansión romana, para lo cual centró su atención en fuentes y análisis comprensibles desde un enfoque de Roma como agente unificador ${ }^{7}$. Centrada en la colonización, estableció la relación entre la construcción de las provincias y la integración social y cultural de los indígenas, refiriendo el vínculo con la identidad. Esta última, concebida en términos esencialistas, se definía desde el ámbito jurídico, entendiendo las normas legales como su factor de promoción. Ser romano suponía vivir de acuerdo a las leyes de una comunidad cívica constituida por la Urbs. Bajo estas consideraciones, la expansión jurídica romana devendría, a la vez que en la transformación política de los espacios públicos nativos, en el cambio cultural de sus habitantes. En definitiva, una visión que consideraba, junto a la expansión política de Roma, la de su cultura.

Como señala Freeman ${ }^{8}$, el supuesto fundamental de la romanización clásica consideraba la colonización como proceso efectivo de integración de comunidades nativas a la sociedad romana, lo que habría permitido construir un imperio de dimensiones inalcanzables por la sola fuerza de las armas y sin la aceptación de los conquistados. Después de todo, las evidencias históricas apoyaban esta perspectiva. La vastedad del imperio romano suponía, al menos en principio, la adhesión de sus miembros. La construcción de las provincias y el consecuente desarrollo de un sistema administrativo, económico, jurídico, militar y lingüístico serían factores para que los conquistados se identificaran culturalmente con el poder central, lo que devendría en una transformación total. No se trataba, sin embargo, de un cambio espontáneo ni surgido por iniciativa local, sino de una política romana promovida con esos fines ${ }^{9}$. La Urbs, como agente transformador, se concebía como responsable de la incorporación nativa en lo que podemos identificar, siguiendo la crítica de Wulff $^{10}$, como un proceso de substitución identitaria.

Así lo vemos en Theodor Mommsen ${ }^{11}$, precursor de la teoría de la romanización ${ }^{12}$, quien relevaba la capacidad de Roma para la unificación sociocultural de sus miembros a través de

\footnotetext{
${ }^{7}$ Respecto de las fuentes del imperialismo, sus características y alcances véase Wells, Peter. 2004. “The Barbarians Speak: How the Conquered Peoples Shaped Roman Empire", en Champion, Craige (ed.), Roman Imperialism: Readings and Sources, Estados Unidos, Blackwell Publishing, pp. 258-277.

${ }^{8}$ Freeman, Philip. 1997. "Mommsen to Haverfield: the origins of studies of romanization”, en Mattingly, David (ed.). Dialogues in Roman Imperialism, JRASS, №23, Rode Island, Portsmouth, p. 27.

9 Véase Bartel, B., 1980. “Colonialism and cultural responses: problems related to Roman provincial analysis", en World Archaeology, №12, pp. 14-22.

10 Wulff, Fernando. 2007. "Las provincias de Hispania en época Republicana: una reflexión sobre enfoques y perspectivas", en Santos, Juan y Torregaray, Elena (eds.) Laudes provinciarum: Retórica y política en la representación del imperio romano, Vitoria, Universidad del País Vasco, p. 47.

${ }^{11}$ Mommsen, Theodor. 1945. El mundo de los Césares, Fondo de Cultura Económica, México D. F., 1945, passim; esp. Introducción.

12 Cf. Freeman, Philip. 1997. "Mommsen to Haverfield", p. 45; Bancalari, Alejandro. 2005. "Theodor Mommsen, el mundo romano y sus proyecciones: a propósito del centenario de su muerte", en Atenea, № 492, p. 137.
} 
estrategias políticas dirigidas para ello. La acuñación de monedas, la ley y la lengua serían, para el romanista germano, herramientas de cohesión social durante el imperio que habrían colaborado en la difusión y adopción de la cultura romana en el Mediterráneo ${ }^{13}$. Si la institucionalidad política y jurídica de Roma eran los agentes promotores de la identidad, la implementación de dicho modelo habría generado, de manera análoga, identidades romanas a lo largo y ancho de los territorios.

Apoyando y fortaleciendo esta visión, Francis Haverfield, principal promotor de la teoría de la romanización, coincidía con Mommsen al señalar el modo en que Roma, mediante una política de integración, había incluido a las comunidades indígenas de Britania a través de un proceso de transformación cultural. Se trataba de una perspectiva de la romanización como proceso cultural unívoco, constructor de la comunidad romana en las provincias mediante una integración efectiva ${ }^{14}$. No obstante, ello no suponía que el proceso se efectuara de manera idéntica, pues los factores para su desarrollo se comprendían adaptables a diversas circunstancias; mas devenían en un resultado común: la conversión a la romanitas. De este modo, las identidades locales se asimilarían a la romana de manera plena, cuya connotación absoluta permitiría, incluso, evaluar el éxito de la romanización identificando el nivel de pervivencia del "nacionalismo indígena y la actitud de los pueblos sometidos al poder central"15.

En esta perspectiva, los principales factores de romanización se vinculaban al despliegue de modelos administrativos y jurídicos en las provincias, que habrían promovido y obligado la vinculación entre los nativos y Roma. La aplicación de la ley romana contribuiría al ordenamiento de la sociedad civil, obligando a los nativos a comunicarse en latín y, junto con ello, a incorporarse en prácticas políticas y jurídicas características de la Urbe, especialmente a las élites locales. Asimismo, la organización del ejército conformaría otra vía de transformación identitaria, puesto que, luego de las disputas de la conquista, la incorporación de locales a la milicia romana brindaría espacios para, por un lado, conocer las características políticas de la romanidad, y, por otro, establecer vínculos bajo un código simbólico-militar común que habría contribuido a la identificación con el poder central.

\footnotetext{
${ }^{13}$ Los estudios de Mommsen refieren, dentro de las estrategias de la estabilidad romana, cierto nivel de tolerancia conveniente con prácticas culturales locales. Sin embargo, su propuesta se basa en el carácter transformador de Roma, comprendida por la irradiación de los fundamentos de su civilización. Por ello, si bien sus obras evidencian características de los pueblos indígenas, estas se comprenden en la medida que resultan coherentes con el sistema social y político romano, y que permite entender cómo la Urbe influyó en todo el orden provincial, construyendo una cultura vinculante en el extenso territorio del imperio. Véase Roces, Wenceslao. 1945. "Prólogo", en Mommsen, Theodor. El Mundo de los Césares, México D. F., Fondo de Cultura Económica, p. 16.

${ }^{14}$ Haverfield, Francis. 1915. The Romanization of Roman Britain, London, Oxford University Press, pp. 9-22.

${ }^{15}$ Alonso-Nuñez, José. 1989. "Reflexiones sobre el imperialismo romano en Hispania", en Studia Histórica. Historia Antigua, №7, pp. 7-8.
} 
En definitiva, el desarrollo de la identidad romana sería resultado de la construcción de espacios cívicos que, sin más, representaban a la Roma misma en las provincias. Por ello, como ilustrativamente expone Harris a propósito de la identidad y la expansión, las ciudades debían organizarse a "imagen y semejanza" ${ }^{16}$ de la Urbe. Esta forma de vida, seductora en tanto civilizada, se habría transformado en un modelo atrayente a las comunidades indígenas, que apartarían sus características sociales definitorias para adherirse a las formas romanas de vinculación social.

La visión que referimos, que constituye el fundamento de la teoría clásica, se sustenta en el principio de superioridad cultural romana por su condición civilizada, cuya superposición ante pueblos culturalmente inferiores se consideraba un beneficio ${ }^{17}$; cuestión promovida por los romanos respecto de su imperialismo como ejercicio legítimo. Junto a la política se entregaría "una cultura superior y seductora" ${ }^{18}$ que llevaría a los indígenas al traspaso entre barbarie y civilización ${ }^{19}$.

Ahora bien, el sentido preeminente de Roma y el alcance totalista de sus transformaciones constituyen aristas profundamente criticadas, principalmente desde los enfoques subalternos y nativistas promovidos por las teorías postcoloniales ${ }^{20}$. El esencialismo jurídico con que la teoría clásica define la cultura romana ha sido criticado por otorgarle un carácter estático; situación que, desde el análisis a la cultura material, resulta incoherente. Los vestigios evidencian influencias culturales bilaterales promovidas en fases dinámicas que posibilitaron, con la intervención y decisión de las comunidades, conformar escenarios de comunicación entre las sociedades colonizadas y el poder central ${ }^{21}$.

\footnotetext{
${ }^{16}$ Harris, William. 2002. "Roma vista desde afuera", en: Semana de estudios romanos, Vol. XI, p. 52.

${ }^{17}$ Véase Hingley, Richard. 2000. Roman Officers and English Gentlemen. The Imperial Origins of Roman Archaeology, Routledge, London-New York, pp. 4-15.

${ }^{18}$ Bancalari, Alejandro. 2007. Orbe romano, p. 69.

${ }^{19}$ En este marco, las ideas de Mommsen sobre la guerra justa influenciaron profundamente los estudios posteriores del imperialismo, que tuvieron directa responsabilidad en el ideario de la romanización como proceso civilizador. Así podemos verlo en Harris (Harris, William. 1979. War and Imperialism in Republican Rome, USA, Oxford Universisty Press, passim), quien -si bien niega el carácter defensivo del imperialismo promulgado por Mommsen- estableció en el imperialismo la promoción del ideario cívico romano en las provincias, propuesta que luego vincularía con la identidad en términos de incorporación ciudadana. Perspectiva esta última que, aun desde una mirada más crítica, vemos en Bauman, R. A. 2000. Human Rights in Ancient Rome, Londres-Nueva York, Routledge, pp. 36 y ss., esp. 87110; Woolf, Greg. 1998. Becoming Roman. The origins of provincial civilization in Gaul, Cambridge, Cambridge University Press, pp. 54-57.

${ }^{20}$ Freeman, P. 1997. “Mommsen to Haverfield”, p. 28; Barrett, John. 1997. "Romanization: a critical comment", en Mattingly, David (ed.). Dialogues in Roman Imperialism, p. 51.

${ }^{21}$ Wallace-Hadrill, Adrian. 2000. "The roman revolution and material culture", en Paschoud, Francois; Paschoud, Francois; Giovanni, Adalberto y Grange, Bernard (eds.). La Revolution Romaine après Ronald Syme: Bilans at perspectives, Entretiens sur l'antiquité classique, Geneva, Vandoeuvres, pp. 310-11; Keay, Simon y Terrenato, Nicola (eds.). 2001. "Preface", en Italy and the West: Comparative Issues in Romanization, Oxford, Oxbow, p. IX; Hingley, Richard. 2005. Globalizing Roman Culture: Unity, Diversity and Empire, Londres - New York, Rutledge, p. 55.
} 
Bajo estas consideraciones, el concepto clásico de romanización ha perdido legitimidad. Por lo mismo, y desde una mirada principalmente histórica, se ha propuesto una renovación del proceso de intercambio cultural desarrollado en la conquista. Desde una arista estrictamente política y transversal se ha derivado en una mirada más flexible y conciliadora, cuya presencia, aunque debatida, entrevemos en el análisis historiográfico actual.

\section{Hacia una nueva romanización}

El juicio negativo propiciado a la teoría de la romanización ha conllevado la transformación del concepto. Desde una visión hegemónica y unilateral, la crítica ha permitido entenderla como proceso de diversidad y de cambio, caracterizado por su desarrollo en función de las particularidades de las comunidades locales ${ }^{22}$. A este respecto, Woolf ${ }^{23}$ explica que no habría solo una forma de romanización, sino diferentes experiencias entre conquistadores y conquistados, quienes aportaron aspectos culturales a la sociedad romana en las provincias; planteamiento que comparten Torelli ${ }^{24}$, Keay y Terranato ${ }^{25}$, y, parcialmente, Barrett ${ }^{26}$.

La renovación del concepto, que considera el espectro local, ha promovido intentos por explicar la romanización de manera bilateral. Las propuestas de Millet ${ }^{27}$, importante estudioso de Britania, constituyen buen ejemplo, puesto que incorporan a las élites locales como agentes de la romanización. De acuerdo con Millet, el sistema político romano habría propiciado que las elites ansiaran su incorporación a la Urbs, generándose, en esta instancia, lo que se comprende como un proceso de romanización autónomo; que más tarde, de manera similar, sería referido por Woolf ${ }^{28}$, en el caso de la Galia, como fase de emulación. Se trata de

\footnotetext{
${ }^{22}$ Woolf, Greg. 1997. "Beyond Roman and Natives”, en World Archaeology, Vol. 28, №3, pp. 339-350.

23 Íbid., pp. 341-344.

24 Torelli, Mario. 1995. Studies in the romanization of Italy, Canadá, University of Alberta Press, pp. 2-15.

${ }^{25}$ Keay, Simon y Terrenato, Nicola (eds.). 2001. Italy and the West. p. 113; véase también Prosdocimi, A. (ed.). 1978. Popoli e civilità dell'Italia antica, Roma, Biblioteca di Storia Patria, passim.

${ }^{26}$ Cf. Barrett, John. 1997. "Romanization: a critical comment”, p. 53.

27 Millet, Martin. 1990. The Romanization of Britain: an essay in archaeological interpretation, Cambridge, Cambridge University Press, passim; Millet, Martin. 2003-2004. "The Romanization of Britain: changing perspectives", en Kodai: Journal of Ancient History, №13-14, pp. 169-173.

${ }^{28}$ Woolf, Greg, 1998. Becoming Roman, pp. 54-76. Plantea el modo en que el sistema de administración romano permitió, a través del ejercicio de los cargos públicos, ofrecer un modelo a imitar por los indígenas de la Galia, quienes terminaron por incorporarse a las formas de vida romana en un proceso de emulación. No habría una conversión abrupta sino paulatina, surgida desde el interior de las provincias en su espíritu por imitar las formas de vida romana que, en último término, habrían conllevado el abandono de sus prácticas originarias. Se trata de una propuesta en la que la romanización habría sido objeto de un proceso voluntario y en la que las élites provinciales habrían propiciado la incorporación romana en función de los beneficios aparejados a dicha condición de romanidad. En la misma línea había interpretado el fenómeno Sherwin-White, Adrian. 1973. The Roman Citizenship, Oxford University, pp. 222-224; véase también Brunt, P. A. 1976. "The Romanization of local ruling classes in the roman empire", en Pippidi, D. M. (ed.), Assimilation et résistance à la culture grecorromaine dans le monde ancien, Les Belles Lettres, Paris, pp. 161-174.
} 
propuestas que, aun cuando sitúan a Roma en la cabeza de la relación con los nativos, destacan la decisión de las autoridades locales como factor de integración, lo que ha abierto el debate hacia enfoques que aluden la transferencia bidireccional de cultura. Desde esta perspectiva, los encuentros culturales no se entienden desde visiones impositivas, por cuanto la vinculación entre individuos, aun en escenarios bélicos, supone un esfuerzo por establecer relaciones sociales que derivan en el encuentro y la transferencia cultural. En este contexto entendemos los esfuerzos de Terrenato por substituir el concepto de romanización por el de "bricolage cultural" ${ }^{29}$, y de Webster, por su parte, por el de "criollización" ${ }^{30}$, toda vez que adquieren sentido en una nueva construcción identitaria definida por la confluencia de las culturas locales y la central.

Más allá del denominativo, el enfoque contemporáneo de la romanización apunta que, si bien la vinculación entre Roma y las sociedades indígenas se desarrolló a partir de las características locales, el despliegue de un modelo provincial igualmente estableció el vínculo identitario entre los nativos y el poder central. No existiría, pues, una forma única de romanizar, sino una política de tolerancia con las comunidades que propiciaría su transformación a la romanidad mediante la paulatina adopción de sus características.

Los diferentes escenarios de encuentro, incluso en la Península Itálica, han promovido el estudio de la romanidad a menor escala y en función de los problemas específicos de los pueblos en contacto. Gabba ${ }^{31}$ y Torelli ${ }^{32}$ coinciden al señalar que las diferencias de los pueblos de Italia conllevaron distintas vías de aproximación romana, que si bien fueron aunadas bajo una identidad en referencia con la Urbs, ello no supuso el completo abandono de sus características anteriores al encuentro, lo que ha relevado la necesidad por comprender la romanización y la construcción de la identidad en términos holísticos. Algunos esfuerzos interesantes $\mathrm{y}$, por cierto, sugerentes, resultan de la aplicación de categorías de análisis comparadas, como ocurre con la idea de interconexión del mundo romano en términos

\footnotetext{
${ }^{29}$ Terrenato, Nicola. 1998. "The Romanization of Italy: Global Acculturation or Cultural Bricolage?”, en Forced, C., Hawthorne, J. y Witcher, R. (eds.), Theoretical Roman Archaeology Journal, №97, 1998, pp. 20-27.

${ }^{30}$ Webster, Jane. 2001. “Creolizing the Roman Provinces", American Journal of Archaeology, №105, pp. 209-225.

${ }^{31}$ Gabba, Emilio. 2000. "El problema de la "unidad" de la Italia romana", en Gabba, Emilio y Laffi, Umberto, Sociedad y política en la Roma Republicana, Pacini, Pisa, pp. 11-24.

32 Cf. Torelli, Mario. 1995. Studies in the romanization of Italy, p. 13. La perspectiva de Torelli a este respecto es sumamente interesante, pues si bien concluye que por los análisis de la cultura material hubo niveles de homogeneización cultural propiciados por la romanización, ello no permite entender Italia como realidad unívoca. Las propias características de la Italia anterior a la expansión romana demuestran gran disparidad cultural entre los diversos pueblos allí asentados, lo que se evidencia incluso en la carencia de sistemas comerciales comunes. El área itálica se correspondería más bien a un concepto lingüístico que a formas comunes de sociedad o cultura.
} 
globales. Así lo vemos en Bancalari ${ }^{33}$, quien, en coherencia con Sweetman ${ }^{34}$, Hitchner ${ }^{35}$ y Witcher ${ }^{36}$, promueve el análisis de la romanización en códigos de globalización. El principio que sustenta esta visión es que si bien el avance romano por el orbe estableció estrategias de integración desde el poder central, estas tuvieron la flexibilidad para adaptarse e incorporar las características locales de las provincias, deviniendo en la construcción de una cultura común, promotora de una identificación con Roma como entidad universal. Así, Bancalari alude la romanización-globalización como "un macro proceso y un conjunto de acciones concretas que tienden a una asimilación, a una cierta identidad y a principios comunes de diversos pueblos que componen un mundo interconectado [...] una integración y unidad -a escala planetariadentro de una diversidad" ${ }^{37}$. Asimismo, y como también hiciera Woolf ${ }^{38}$, esta visión destaca el rol de las élites, cuya incorporación en el sistema de administración de las provincias habría facilitado la relación entre las sociedades provinciales y el poder central. Bien promoviendo la incorporación de sus comunidades, bien sirviendo como ejemplo a imitar, las élites habrían colaborado en la construcción de "una identidad común entre los miembros del imperio"39.

Se trata sin duda de propuestas interesantes, aunque no parecen destacar lo suficiente el carácter multidimensional de la identidad, ni los factores específicos de las comunidades locales en la construcción de identidades coherentes con sus prácticas sociales ${ }^{40}$. Son modelos que, en definitiva, comprenden a la urbe como el agente coordinador de las identidades locales y de Roma como promotora de una cultura atractiva.

Con todo, las teorizaciones de la romanidad bajo la perspectiva de la globalización resultan útiles para comprender un modelo de diversidad unificada, ya que posibilitan ubicar las características locales dentro del universo en que se desarrolló la política romana; y permiten, además, entender el proceso de conquista más allá de la belicosidad y la imposición. No obstante, y porque su interés es principalmente el ámbito político, el concepto no ahonda en las modalidades que diferenciaron la identidad en el territorio romano, ni tampoco las formas de interacción local que pudieron afectar en la construcción de una identidad común. Ello, por

\footnotetext{
33 Bancalari, Alejandro. 2007. Orbe romano, pp. 89 y ss.

${ }^{34}$ Sweetman, R. 2007. "Roman Knossos: The Nature of a Globalized City", en American Journal of Archaeology, Vol. 111, №1, pp. 61-81.

${ }^{35}$ Hitcher, Bruce. 2007. The First Globalization: The Roman Empire and Its Legacy in the 21st Century, Oxford, Oxford University, passim.

${ }^{36}$ Witcher, R. 2000. "Globalization and Roman Imperialism: perspectives on identities in Roman Italy", en Herring, E. y Lomas, K. (eds.). The Emergence of State Identities in Italy in the First Millenium BC., Londres, Accordia Research Institute, pp. 213-225.

${ }^{37}$ Bancalari, Alejandro. 2007. Orbe romano, p. 90.

${ }^{38}$ Woolf, Greg. 1998. Becoming roman, pp. 42 y ss.

${ }^{39}$ Bancalari, Alejandro. 2007. Orbe romano, p. 27.

${ }^{40}$ Véase Revell, Louise. 2016. Ways of Being Roman, Oxford-Filadelfia, Oxbow Books, pp. 14-18.
} 
cuanto constituye la conceptualización de un proceso generalizado por el Mediterráneo, cuyo propósito es, precisamente, comprender las convergencias asumiendo las divergencias. Y es, precisamente, a este aspecto divergente, al que apuntan los estudios de la etnicidad.

\section{Etnicidad}

Durante los últimos 50 años, la etnicidad ha constituido uno de los enfoques más influyentes en el análisis de la identidad romana. Liderados por la escuela arqueológica británica, los estudios fundados en esta perspectiva se interesan por la cultura material, y su propósito es comprender las características específicas de las comunidades adheridas a Roma en su proceso de expansión.

Rechazando la noción moderna de la identidad, promueven una nueva conceptualización de la misma, fundada en visiones contemporáneas y, como señalamos, directamente vinculadas con las teorías de la descolonización. En este marco, los estudios de la etnicidad centran su atención en aquellos factores que, permitiendo la identificación entre los miembros de un grupo étnico, construyen comunidad; perspectiva que, aplicada al escenario romano, ha conllevado el reconocimiento de las características específicas de las comunidades dominadas por Roma. Los conceptos de etnicidad y de grupo étnico, empero, son imprecisos, pues se utilizan para referir diversos aspectos sociales. Además, como resultado de paradigmas postcoloniales, su espíritu reivindicador los ha cargado de emocionalidad ${ }^{41}$, por lo que no carecen de ideologías y preconcepciones que, en los estudios romanos, los han llevado a ser utilizados con propósitos de constatación, resultando, en ocasiones, en análisis acomodaticios o tan extremados como aquellos que reprochan.

Si consideramos el criterio de la identidad y sus modalidades de construcción, la etnicidad ha adquirido significado como proceso y como resultado. Como proceso refiere los aspectos que inciden en la construcción de la identidad étnica; como resultado, aquello que permite a una colectividad identificarse como grupo cultural. En este sentido, el concepto de etnicidad se propone como reemplazo al de identidad, puesto que, por una parte, lo contiene, y, por otra, lo aplica a comunidades específicas. No se trata, sin embargo, de definiciones unívocas, pues los diferentes criterios para referir un grupo étnico generan desacuerdo. A este respecto, los principales aportes han surgido desde las ciencias sociales en referencia a procesos históricos contemporáneos, cuyas categorías conceptuales se han aplicado al mundo antiguo. Los análisis más relevantes dan cuenta de los múltiples aspectos que conlleva la formación de un grupo

\footnotetext{
${ }^{41}$ Nash, Manning. 1989. The Cauldron of Ethnicity in the Modern World, Chicago, University of Chicago Press, p. 1. Señalando, precisamente, que la etnicidad la encontramos "among the most complicated, volatile and emotionally charged words and ideas in the lexicón of social science".
} 
étnico y, por consiguiente, que constituyen la etnicidad. Así, mientras que, para Weber ${ }^{42}$, se trata de aspectos comunes en ideología, creencias y cultura material, para Smith ${ }^{43}$ resultan de principal influencia el lenguaje, la ascendencia y el territorio. Por su parte, Nash ${ }^{44}$ destaca la importancia del pasado, de la nacionalidad e incluso de la herencia biológica; y Bartlett ${ }^{45}$ apunta, por sobre todo, las costumbres y las leyes. Planteamientos que, sin duda, permiten comprender la complejidad del concepto.

No obstante su diversidad, los múltiples enfoques de la etnicidad han decantado en la construcción de dos corrientes teóricas de principal influencia, a saber, primordialista e instrumental ${ }^{46}$. Sus diferencias en torno a los factores objetivos y subjetivos que constituyen la etnicidad suscitan discusión respecto del modo en que la percepción de la identidad étnica se vincula con las prácticas culturales, con las relaciones sociales y con las necesidades colectivas ${ }^{47}$.

La corriente primordialista pone énfasis en los lazos de consanguinidad ${ }^{48}$ que afectan en el desarrollo de la identidad, cuyos elementos constitutivos son la tradición, los valores, la religión y la historia. Destacando la importancia del parentesco, explica que los individuos reciben la

42 Weber, Max. 1978. Economy and Society. An Outline of Interpretative Sociology, Berkeley-Los Angeles-Londres, University of California Press, pp. 385-397. Destaca específicamente la creencia en un antepasado común, el lenguaje, los rituales, la concepción compartida de lo que es apropiado y un conjunto de aspectos de la vida cotidiana que construyen códigos: estilos de vestimenta, tipo de alojamiento, hábitos de comida y bebida y división del trabajo. Las categorías de Weber, sin duda pioneras en su época (1922), constituyeron la base desde la cual se fundarían los análisis de las etnias desde la sociología y los estudios antropológicos.

${ }^{43}$ Smith, Anthony. 1986. The Ethnic Origins of Nations, Oxford, Blackwells, passim. Establece la etnicidad como las características esenciales del grupo étnico, cuya formación y definición se desarrolla a partir de un conjunto de categorías que permiten desarrollar comunidad: nombre del grupo conocido por todos, mito de origen, historia compartida y conocida, cultura distintiva, lengua, religión, asociación con un territorio y sentido de solidaridad.

${ }^{44}$ Nash, Manning. 1989. The Cauldron of Ethnicity, pp. 5-6. En cuanto a los aspectos biológicos, considera el cuerpo, que poseería la herencia genética traspasada por generaciones anteriores, lo que responde a una visión de la etnicidad que supone cierta rigidez, por cuanto existiría cierto nivel de determinismo. No se trata de un factor unívoco, pero, de acuerdo con Nash, sería relevante. Por su parte, también considera el lenguaje, los orígenes comunes, la historia compartida y la nacionalidad. Esta última no se entiende en términos "modernos", sino en tanto la relación de una comunidad con un territorio definido, incluso en un plazo de ocupación breve.

45 Bartlett, Robert. 1994. The Making of Modern Europe. Conquest, Colonization and Cultural Change, Inglaterra, Penguin, pp. 197-220. Propone la etnicidad como el resultado de un vínculo entre los miembros de una colectividad vinculada por un grupo de costumbres, la existencia de una ley que ordena los contactos entre la comunidad y que liga a sus miembros entre sí, y, finalmente, una lengua común.

${ }^{46}$ Hyun Jin, Jim. 2009. Ethnicity in Ancient Greece and China, Londres, Duckworth, pp. 6 y ss; Río, Manuel. 2002. "Visiones de la etnicidad", en Revista española de investigaciones sociológicas, Vol. 98, pp. 85-96. Una sucinta pero clara explicación en Comaroff, John y Comaroff, Jean. 2011. Etnicidad S.A., Madrid, Katz, pp. 65-69. Véase también, Jones, Siân. 1997. The Archaeology of Ethnicity, Londres-Nueva York, Routledge, passim.

47 Jones, Siân. 1997. The Archaeology of Ethnicity, p. 65.

48 Shils, Edward. 1957. Center and Perisphery: essays in macrosociology. Selected papers of Edward Shils, Vol. II, Chicago, Chicago University Press, p. 122; Véase también Jones, Siân. 1997. The Archaeology of Ethnicity, p. 64; Arno, Claudia. 2012. How Romans Became "Roman": Creating Identities in an Expanding World, Tesis Doctoral, Michigan, Universidad de Michigan, pp. 12-16. 
identidad de sus padres en un proceso del que se hacen parte de manera inconsciente, a causa de una necesidad psicológica natural del hombre por sentirse parte de un grupo ${ }^{49}$. Así, cual carga genética, la identidad étnica se torna heredable y los nuevos miembros de una comunidad la adquieren más bien producto del parentesco que de necesidades prácticas ${ }^{50}$. Se trataría de una suerte de identidad base, adquirida desde el nacimiento a través de espacios y mecanismos destinados para ello por la misma comunidad ${ }^{51}$. De este modo, la corriente primordialista concibe la identidad étnica como la más natural en el hombre, pues se desarrollaría en los apegos sociales primarios del individuo ${ }^{52}$.

Si bien nos encontramos ante una concepción que no omite del todo la raíz sociocultural de la identidad, no releva su importancia como factor primordial en la formación, el traspaso y el cambio de la misma. Por el contrario, establece más bien una condición estática de la etnicidad, refiriéndola en términos casi inmutables y puros. Ideas que, precisamente, constituyen el foco de reprobación de la corriente instrumental ${ }^{53}$.

En efecto, las críticas de la corriente instrumental advierten que la concepción de la identidad en términos inmóviles es absurda, pues supone asumir que las transformaciones sociales no afectan el modo en que las comunidades se autoconceptualizan. Los cambios históricos conllevan modificaciones en la convivencia, en la relación entre las etnias y en las necesidades del devenir que afectan directamente en el modo en que los individuos se entienden a sí mismos y a sus comunidades; problemas que, omitidos por la corriente primordialista, la invalidan para explicar las variaciones de la identidad en procesos de mixtura étnica ${ }^{54}$. En este sentido, para la crítica instrumental la concepción pura e inmutable de la identidad constituye una idealización, ya que no responde a procesos históricos reales, sino a simplificaciones y generalizaciones que no reparan en las circunstancias específicas que afectan en su conformación y cambio en cada grupo étnico ${ }^{55}$. Así, destaca la naturaleza móvil de la identidad y reconoce en los ámbitos social, político y económico sus principales factores de

\footnotetext{
49 Isaacs, Harold. 1974. "Basic group identity: ideals of the tribe”, en Ethnicity, Vol. 15, № 1, pp. 27-30.

50 Véase Geertz, Clifford. 1963. "The integrative revolution: primordial sentiments and civil politics in the new states", en Geertz, C. (ed.). Old Societies and New States, The Free Press, New York, p. 108-110.

${ }^{51}$ A este respecto, advierte Kellas: "Identity and behavior are partly genetic, but they are also shaped by context and choice [...] Human nature provides the necessary conditions for ethnocentric behavior, but politics converts this into the 'sufficient conditions' for nationalism as we understand it today". Kellas, James. 1991. The Politics of Nationalism and Ethnicity, Londres, Macmillan, p. 19.

52 Isaacs, Harold. 1974. "Basic group identity", p. 30; Keyes, Charles. 1976. "Towards a new formulation of the concept of ethnic grup", en Ethnicity, №3, pp. 202-213.

${ }^{53}$ Cf. Barth, Fredrik. 1969. "Introduction", en Barth, F. (ed.). Ethnic Groups and Boundaries, Boston, Little Brown, p. 10.

54 Véase Arno, Claudia. 2012. How Romans Become Roman, p. 14.

55 Íbid., pp. 12-16; Jones, Siân. 1997. The Archaeology of Ethnicity, pp. 54-79.
} 
desarrollo. Los cambios en estos últimos afectan a los individuos que -de forma conscienteordenan, dan sentido y establecen los límites de la comunidad; proceso que, junto con definirlos, establece los límites con otros grupos étnicos.

De acuerdo a esta perspectiva, un grupo étnico no solo es resultado de tradiciones o valores -que, por cierto, no se excluyen-, sino también de necesidades políticas o económicas comunes, indispensables de salvaguardar, que guían el actuar ${ }^{56}$. A este respecto, Cohen advierte: "los grupos étnicos no son simplemente la suma total de todos sus miembros individuales, y su cultura no es la suma total de estrategias adoptadas por individuos independientes. Normas, creencias y valores son efectivos y tienen su propio poder de limitación solo porque son las representaciones colectivas de un grupo y son respaldados por la presión de ese grupo" ${ }^{57}$. De este modo, quienes forman parte de esa comunidad no pueden transformar arbitrariamente sus componentes, sino que "deben pagar el precio de esa membresía de participación de un grupo de actividades simbólicas por una medida de adhesión a los objetivos del grupo" ${ }^{158}$.

Aun cuando la perspectiva instrumental permite referir la incorporación de nuevos miembros, explicar confluencias culturales y relevar tanto la conciencia del individuo como los factores de conveniencia en la construcción de la identidad, la visión extremada que adquieren sus presupuestos dificulta comprender la influencia de estructuras más estables de la comunidad-como la familia, las instituciones y la tradición-, que sin duda adquieren relevancia en los procesos de construcción social. En este sentido, el debate entre ambas corrientes da cuenta de la necesidad de encontrar factores comunes que permitan entender el desarrollo de los grupos étnicos desde perspectivas complementarias ${ }^{59}$. Después de todo, ningún grupo étnico actúa únicamente por ideales, ni tampoco solo por propósitos específicos. Ambas dimensiones confluyen de manera natural ${ }^{60}$.

En este contexto, el estudio de la etnicidad en el mundo romano ha generado una interesante labor de mixtura. Por un lado, ha promovido el análisis de las comunidades indígenas desde categorías y circunstancias más estables centradas en los grupos étnicos antes de su contacto con Roma. Por otro lado, ha propiciado su examen en procesos de cambio a través del estudio de las respuestas o efectos de la conquista en dichos grupos.

Un interesante y, ciertamente, notable esfuerzo por definir la etnicidad dejando atrás la dicotomía intrumental-primordialista ofrece Siân Jones en su obra The Archaeology of Ethnicity, de gran influencia en los últimos años. Asumiendo los problemas definitorios del concepto de

\footnotetext{
56 Jones, Siân. 1997. The Archaeology of Ethnicity, p. 74.

57 Cohen, Abner. 1974. Urban Ethnicity, Londres, Tavistock Publications, 1974, p. XIII.

${ }^{58}$ Idem.

${ }^{59}$ Véase, por ejemplo, McKai, James. 1982. "An exploratory synthesis of primordial and mobilizationist approaches to ethnic phenomena", en Ethnic and Racial Studies, Vol. 5, pp. 401-402.

60 íbid., p. 403; Jones, Siân. 1997. The Archaeology of Ethnicity, p. 80.
} 
etnicidad, promueve su aplicación a la antigüedad romana bajo una mirada fundada, principalmente, en la antropología, reprobando las clasificaciones sociológicas tradicionales de las sociedades en función de sus estadios de desarrollo cultural. De acuerdo con Jones, la etnicidad permite comprender cabalmente las características de los grupos étnicos, ya que vincula los factores objetivos y subjetivos que atañen en su conformación y devenir. En cuanto a los primeros, la etnicidad se refiere al resultado de la objetivación de practicas culturales convertidas en hábitos. Los segundos, por su parte, aluden a la manera en que estas características afectan en los sujetos de un grupo ${ }^{61}$. De este modo, define la entnicidad como "todos los fenómenos sociales y psicológicos asociados a una identidad de grupo culturalmente constituido [...] se centra en las formas en que los procesos sociales y culturales se cruzan entre sí en la identificación de, y la interacción entre, los grupos étnicos"62.

En definitiva, una explicación que permite contemplar el amplio espectro de la etnicidad, cuya heterogeneidad encontramos en los principales estudios de la historia romana.

\section{Etnicidad en Roma: de la resistencia a la identidad discrepante}

Aun cuando los estudios de la etnicidad conforman un cuadro heterogéneo, buena parte convergen en el rechazo a los conceptos y explicaciones de la historiografía tradicional ${ }^{63}$ y en promover, desde un enfoque nativista, una interpretación de reivindicación étnica. Contrarios al imperialismo, los análisis de la etnicidad impugnan la romanización en términos conceptuales e históricos. En cuanto al concepto, aducen su carácter contemporáneo, cargado de prejuicios, ideologías y motivaciones políticas que no permiten entender los alcances de la expansión romana. Así lo expresan Mattingly ${ }^{64}$, Webster ${ }^{65}$, James ${ }^{66}$ y Hingley ${ }^{67}$, que no solo sugieren la utilización de otros términos para explicar los efectos de la conquista, sino que exponen los problemas derivados de asumir las categorías de la romanización para el conocimiento de sociedades que, erróneamente, se consideran dominadas.

\footnotetext{
61 Jones, Siân. 1997. The Archaeology of Ethnicity., pp. 51-52; 128.

62 Ibid., p. XIII.

63 Por historiografía tradicional entendemos toda aquella producción literaria sustentada en la teoría de la romanización. Primero en su versión clásica; luego, aunque en mayor diálogo con las corrientes de ruptura, las visiones más flexibles y contemporáneas.

64 Mattingly, David. 2002. "Vulgar and weak 'Romanization' or time for a paradigm shift", en Journal of Roman Archaeology, №15, pp. 536-540.

${ }^{65}$ Webster, Jane. 2001. “Creolizing the Roman Provinces, pp. 209-225.

66 James, Simon. 2001. "Romanization and the peoples of Britain", en Keay, Simon y Terrenato, Nicola (eds.). Italy and the West, pp. 187-209.

${ }^{67}$ Hingley, Richard. 1997. "Resistance and domination: social change in Roman Britain", en Mattingly, David. (ed.). Dialogues in Roman Imperialism. pp. 87-96.
} 
Desde esta perspectiva, la crítica declara, en primer lugar, que la connotación totalizante y civilizadora de la romanización otorga una visión de inferioridad en los pueblos conquistados, inconscientes de su propia identidad. En segundo lugar, que la suposición de la romanización como proceso de aculturación no permite entender el rol de los nativos en la interacción y negociación de su identidad con Roma ${ }^{68}$. Finalmente, y en tercer lugar, que la romanización implica asumir la incapacidad de reacción indígena ante Roma, así como también su influencia en las comunidades romanas provinciales. Así, rechazan el uso del término, que implicaría asumir una condición peyorativa y homogénea en las culturas conquistadas; cuestión que, arguyen, de acuerdo con las fuentes, resulta imposible afirmar.

En esta línea, la etnicidad niega la homogeneización cultural en el Mediterráneo, aduciendo que las sociedades del territorio romano ofrecen pruebas de lo contrario. El análisis principalmente arqueológico- revela diferencias entre las comunidades locales y la cultura central, incluso en zonas occidentales del imperio cuya influencia debería ser, en principio, mayor ${ }^{69}$.

Rechazando las fuentes utilizadas por los estudios de la romanización, expresan la necesidad de analizar la conquista desde la cultura material, a partir de la cual reconocen la identidad nativa en sus diferentes fases de contacto con la Urbs. Así, niegan un proceso romano de aculturación, que propugnan como una construcción historiográfica, pero históricamente irreal. En esta línea encontramos a Torelli ${ }^{70}$, Haselgrove ${ }^{71}$ y Bénabou ${ }^{72}$, quienes, además, exponen los objetivos económicos de la construcción provincial, refutando la misión civilizadora asignada por la tradición. Por lo demás, la interpretación de la romanización clásica se refiere

\footnotetext{
68 Véase Jones, Siân. 1997. The Archaeology of Ethnicity, p. 135; Twedie, Fiona. 2015. "Volterrae and the Gens Caesina", en Roselaar, Saskia (ed.). Process of Cultural Change and Integration in the Roman World, Leiden-Londres, Brill, pp. 92-106; Scopacasa, Rafael. 2015. "An Alllied of Integration: Italian Elites and Consumption in the Second Century B. C.", en Roselaar, Saskia (ed.). Process of Cultural Change, pp. 39-56.

${ }^{69}$ A partir de un análisis de los territorios occidentales del imperio, en esta línea encontramos a: Barrett, John. 1997. "Romanization: a critical comment, p. 51; Fear, Andrew. 1996. Rome and Baetica: Urbanizarion in Southern Spain c.50 BC-AD 150, Oxford, Clarendon Press, pp. 230 y ss; Alcock, Susan. 1997. "The problem of romanization, the power of Athens", en Hoff, Michael y Rotroff, Susan (eds.). The Romanization of Athens, Oxford, Oxbow, p. 1; Beltrán, Francisco. 1999. "Writing, language and society: Iberians, Celts and Romans in Northeastern Spain in the 2nd and 1st centuries B. C.", en Bulletin of the Institute of Classical Studies, №43, pp. 131-132; Crawley, Josephine. 2003. "Roman Africa", en Merryweather, Andrew y Prag, Jonathan (eds.). Digressses, Londres, University of London, pp. 7-32; Mattingly, David. 2002. "Vulgar and weak Romanaization", pp. 536-540; Woolf, Greg. 2001. "The Roman cultural revolution in Gaul", en Keay, Simon y Terrenato, Nicola (eds.). Italy and the West, p. 173; Laurence, Ray. 2001. "Roman narratives: the writing of archaeological discourse - a view from Britain?", en Archaeological Dialogues, № 8, pp. 90-100.

${ }^{70}$ Torelli, Mario. 1995. Studies in the romanization of Italy, 1 y ss.

${ }^{71}$ Haselgrove, Colin. 1984. "Romanization before conquest: Gaulish precedent and British consequences", en Blagg, Thomas y King, Anthony (eds.). Military and Civilian in Roman Britain. Cultural relationships in a frontier province, Oxford, BAR British Academy, Vol. 136, pp. 1-64.

${ }^{72}$ Bénabou, Marcel. 1976. La résistance africane à la romanization, París, Éditions la Découverte, pp. 18-20.
} 
como mecanismo de ahistorización, por cuanto contribuye a ocultar las características específicas de los pueblos nativos ${ }^{73}$. Se trata, en definitiva, de una visión que promueve el estudio de las comunidades desde una mirada local, apartándose de las explicaciones totalistas bajo criterios políticos y/o jurídicos. Sus análisis han permitido comprender la existencia de identidades plurales en contacto con Roma, más allá de aquellas representadas por el poder central ${ }^{74}$.

La diversidad de pueblos conquistados por la Urbe ha llevado a que la investigación se interese en las respuestas de cada comunidad ante Roma, considerando, además, sus segmentos sociales, lo que ha generado nuevos focos de estudio centrados en sus características organizativas y culturales ${ }^{75}$. En esta línea, los análisis arqueológicos ofrecen una interpretación dual de la cultura material indígena: por un lado, como prueba de su conservación identitaria tras la conquista ${ }^{76}$; por otro, como evidencia del rechazo a las costumbres romanas que, en los grupos subalternos, mostraría resistencia a la aculturación. Esta última es, sin duda, una propuesta de suma relevancia, ya que impulsa una mirada de los indígenas como protagonistas y de sus colectividades como entidades organizadas, conscientes de su etnicidad, incluso de manera diferenciada en un mismo grupo. Asimismo, el concepto de resistencia muestra a la Urbs en un rol menos poderoso y pone en evidencia la necesidad de comprender a sujetos que, omitidos por la historiografía tradicional, se consideran imprescindibles para entender el devenir romano.

De cabal importancia resulta señalar este último aspecto, pues el reconocimiento de nuevos actores, conscientes de su rol comunitario, supone cuestionar la construcción del poderío romano, así como también el modo en que el limes se expandió a través de pueblos no siempre en acuerdo con las formas de vida y de funcionamiento de Roma. El rechazo a la romanización conforma un paradigma en sí mismo, que interpreta los cambios en la identidad nativa en función de una posición antirromana de la que habrían resultado identidades discrepantes. El intento de aculturación romano generaría movilizaciones contrarias que, a poco andar, reforzarían la etnicidad local, estableciendo movimientos de rechazo. Britania ${ }^{77}$ y el Norte de

\footnotetext{
73 Mattingly, David. 2011. Imperialism, Power and Identity, Princeton-Oxford, Princeton University Press, p. 204; Hingley, R., "Resistence...", op. cit., p. 96.

${ }^{74}$ Véase Mattingly, David. 2011. Imperialism, Power and Identity, pp. 209-215.

${ }^{75}$ Algunos ejemplos relevantes sobre organización y consecuencias del contacto con Roma en Reece, Richard. 1980. "Town and country: the end of Roman Britain", en World Archaeology, Vol. 12, pp. 77-92; Smith, John. 1997. Roman Villas: A study in Social Structure, Londres, Routledge, passim, esp. 3-20. Ritualidad y cultura en Bénabou, Marcel. 1976. La résistance africane à la romanization pp. 259-262.

${ }^{76}$ Webster, Jane. 2001. “Creolizing the Roman Provinces”, p. 212.

77 Jones, M. y Miles, D. 1979. "Celts and Romans in the Thames Valley: approaches to culture change", en Burnham, Barry y Johnson, Helen (eds.). Invasion and Response: The case of Roman Britain, Oxford, BAR British Series, №3, passim; Hingley, Richard. 1997. "Resistance and domination: social change in Roman Britain", en Mattingly, David
} 
África ${ }^{78}$, lugares en que el avance romano fue más lento y tardío, constituyen localidades representativas, donde el material arqueológico indígena se ha interpretado como fuertemente discrepante ${ }^{79}$.

En un interesante análisis de Britania, Mattingly ${ }^{80}$ plantea que las diferencias sociales constituyeron un factor preponderante en la formación de identidades discrepantes, pues los esfuerzos de aculturación romana se habrían volcado en las capas altas de la sociedad, cuyo poder político y económico constituiría el principal interés del imperio. Allí podríamos encontrar algunos cambios en la identidad, toda vez que la integración en la administración de las provincias pudo contribuir a un acercamiento entre las élites locales y el poder central. No obstante, dichas transformaciones no habrían afectado los segmentos populares, por cuanto su condición subalterna, carente de poder político y económico, no los habría erigido como sujetos atractivos. Además, las diferencias internas de este último grupo habrían generado identidades divergentes incluso antes de la conquista, lo que habría dificultado el despliegue de estrategias de aculturación efectivas. Con todo, Mattingly advierte que el detrimento de la conquista y las dificultades económicas de la vida provincial habrían establecido prácticas sociales que, a poco andar, generarían una identidad común discrepante hacia el poder central.

Si bien en la vastedad del Imperio Romano pudieron existir episodios de discrepancia, el problema de esta mirada es su connotación reivindicativa, que establece concepciones apriorísticas en circunstancias en las que la resistencia no resulta del todo clara. Observamos sus efectos en análisis forzados de las fuentes, en lo que parecieran ejercicios no tanto de interpretación cuanto de constatación ${ }^{81}$. En este sentido, si la romanización clásica constituye,

(ed.). Dialogues in Roman Imperialism, pp. 87 y ss; Mattingly, David. 2011. Imperialism, Power and Identity, pp. 20 y ss.

78 Estudios que nos parecen del todo representativos: Bénabou, Marcel. 1976. La résistance africane à la romanization, passim; Bénabou, Marcel. 1978. "Les Romains ont-ils conquis l'Afrique?", en: Annales: Économies, Sociétés, Civilisation, año 33, № 1, pp. 83-88; Leveau, Philippe. 1978. "La situación coloniale de'IAfrique romaine", en: Annales: Économies, Sociétés, Civilisation, año 33, №1, pp. 89-92.

${ }^{79}$ En esta línea los trabajos de: Hingley, R., “Resistence...”, op. cit, pp. 87-97; Mattingly, David. 2011. Imperialism, Power and Identity, p. 203.

${ }^{80}$ Mattingly, David (ed.). 1997. Dialogues in Roman Imperialism, pp. 204-214.

${ }^{81}$ Buen ejemplo nos parece la reflexión de Adams respecto del bilingüismo y la resistencia a la cultura romana en la Hispania Citerior, que ejemplifica a partir del uso de la lengua vernácula por parte del asesino del legado imperial Lucio Calpurnio Pisón en el interrogatorio efectuado por los soldados romanos. De acuerdo con Adams, las palabras en celtibérico negando su colaboración en la investigación darían cuenta del rechazo por abandonar la identidad lingüística de su cultura, y, a su vez, de resistencia a la cultura romana. Adams, James. 2003. Bilingualism and the Latin Language, Cambridge, Cambridge University Press, pp. 279-280. Coincidimos con Beltrán al considerar este análisis desmesurado, puesto que, tanto las condiciones del interrogatorio -que incluían tortura-, como el carácter individual de la alocución y de la acción, no admiten una interpretación de esa índole. Asimismo, si el carácter de la Bética hubiera sido de resistencia, no resultaría coherente que ese mismo año (25 d.C.) se le hubiera concedido la condición municipal, respuesta, por lo demás, a una petición de la parte interesada. Respecto a la crítica véase Beltrán, Francisco. 2011. “Lengua e identidad en la Hispania Romana", en Paleohispánica, №11, pp. 19-23. 
a ojos de la crítica, el asidero de planteamientos ideologizados y de prejuicios culturales, los análisis de carácter postcolonial no se encuentran ajenos a los mismos, aunque, ciertamente, desde una perspectiva opuesta. Y es que abordar los problemas de la identidad romana supone hacerlo en escenarios de conquista e interacción cultural, ante lo cual, como advirtiera Desideri $^{82}$, no resulta posible abstenerse por completo y no tomar una postura ante el desempeño de Roma.

Ahora bien, la relevancia de este tipo de estudios radica en que han permitido entrever formas de identidad desde planos que superan al político, vinculadas a las prácticas sociales comunes entre individuos que conforman una colectividad. Asimismo, ha relevado nuevos sujetos como agentes de desarrollo identitario más allá de las élites nativas aludidas por las teorías de la romanización, postura con la que se mantiene la tensión. Con todo, el desarrollo de los enfoques culturales, que abordan la identidad desde el discurso y concilian el marco local con el global, han permitido establecer diálogos entre las disciplinas arqueológica e histórica, propiciado nuevas vías para el estudio de la identidad. No se trata de miradas totalmente novedosas, sino de vías complementarias que relevan nuevos componentes de la identidad, así como también de agentes de su desarrollo. Si bien no carece de problemas, nos parece que el enfoque cultural supera, al menos explícitamente, los planteamientos de reivindicación postcolonial.

\section{Identidad cultural}

Desde la década de 1980, el giro cultural en los estudios clásicos ha generado una profunda transformación en los análisis de la romanidad. Como resultado de la crítica postmoderna, ha intentado responder a los problemas de la verdad desde la teoría del discurso, cuya aplicación al contexto romano ha suscitado nuevas formas de abordar la identidad, referida, principalmente, a los individuos en sus diferentes roles sociales.

Desde una mirada holística, construye un espacio de diálogo entre las disciplinas histórica y arqueológica, y, para su conceptualización, considera los diferentes espectros de la vida social bajo una dimensión común, esto es, el discurso. Su connotación simbólica constituye el principal foco de atención, por cuanto refiere el modo en que la sociedad comprende la realidad y la explicita a través de códigos comunes, coherentes y aprehensibles por quienes comparten la cultura ${ }^{83}$. Así, tanto fuentes literarias como cultura material se consideran legítimas para el estudio de la identidad, pues, resultantes de la cultura, remiten a la romanidad ${ }^{84}$.

\footnotetext{
82 Desideri, Paolo. 1991. “La romanizzazione dell'impero”, en Schiavone, Aldo (ed.), Storia di Roma, Vol. 2, Einaudi, Torino, p. 585.

${ }^{83}$ Cf. Habinek, Thomas. 2001. The Politics of Latin Literature, Princeton, Princeton University Press, pp. 3-7.

${ }^{84}$ Véase Gardner, Andrew. 2013. "Thinking about Roman imperialism: postcolonialism, globalization and beyond?", en Britannia, №44, pp. 1-25; Revell, Louise. 2016. Ways of Being Roman, pp. 2-39.
} 
El extenso abanico de la cultura ha desarrollado trabajos sumamente heterogéneos, e, incluso, contradictorios; cuestión que, sin embargo, no constituye un problema a este paradigma. Los múltiples ámbitos y etapas de la vida y los diversos roles sociales, se consideran indispensables para entender la identidad, cuyas diferencias encontramos entre sociedad e individuo de forma recíproca.

Uno de los principales planteamientos del paradigma cultural es que la identidad es dinámica, se transforma a lo largo de la vida, alterando, en consecuencia, el modo en que los individuos se reconocen a sí mismos y al grupo. Aspectos como la edad, el estatus social y económico, el sexo y la ocupación constituyen factores preponderantes de la identidad, que irremediable y naturalmente cambia a lo largo de la existencia individual y colectiva. Así, los discursos de personas y grupos, vinculados con su quehacer y con las estructuras sociales, se entienden como factores con directa injerencia en la construcción y manifestación de la identidad.

Desde estos presupuestos, la mirada cultural propone el análisis de la romanidad a partir de dos escalas. En primer lugar, desde escenarios globales, representados por el marco sociocultural que Roma llevó a las provincias a través de canales vinculantes. Así, los sistemas político, institucional, jurídico, administrativo, económico y urbanístico se consideran vías de primera relevancia. Propugna, en definitiva, un análisis al modelo con que Roma, en su acción universal, construyó una identidad común en todos los territorios bajo su dominio, utilizando para ello diferentes mecanismos de enlace entre el poder central y las provincias. En segundo lugar, se propone el análisis local, aplicado a las comunidades en que estos mecanismos fueron desenvueltos en función de sus particularidades, considerando, además, los aspectos de la cultura provincial traspasados a Roma.

Con todo, los análisis global y local no se conciben de manera apartada, ya que se proponen entender cómo ambas dimensiones se involucraron en la formación de la romanidad en territorios sumamente diversos. Plantea, desde esta perspectiva, el concepto de "glocalización", que alude la construcción dual de la identidad, constituida con aspectos internos, entregados desde la localidad, y externos, aportados por la Urbs. Se trata de un enfoque que, más allá de aculturaciones o rechazos, entiende la identidad romana a partir de confluencias, donde la transferencia cultural establecería las directrices del comportamiento público y privado.

En efecto, los planos privado y público se consideran vitales para entender la identidad, toda vez que el encuentro de ambos supone la conciliación entre el orden social global, constituido por normas de comportamiento esperado, y el ejercicio individual, enmarcado o no dentro de estas. La aprobación o rechazo del desempeño personal en el espacio público sería, mediante respuestas colectivas, mecanismo efectivo de construcción de identidad. El asentimiento 
supondría el reconocimiento como parte de la comunidad; la reprobación, la identificación como foráneo, o, como ha solido designarse, como un otro ${ }^{85}$.

En este marco, el análisis del rol comunitario promovido por la etnicidad se aplica a una pluralidad de sujetos omitidos por las visiones previas y a una menor escala. Ello, por cuanto los sujetos estudiados no responden a colectivos asimilables a una comunidad en su conjunto, sino a segmentos de esta. Así, nuevos sujetos y variables han surgido como objetos de investigación identitaria, como lo reconocemos en el interés por la mujer, los niños y los esclavos, y en temáticas asociadas a estos, como el género, la familia, el cuerpo y la sexualidad ${ }^{86}$.

Si bien todos estos problemas constituyen campos de interés, probablemente el aspecto más significativo de los estudios culturales es su concepción de la identidad como construcción social. Los principales aportes de este enfoque -influido por la teoría de la estructuración de Anthony Giddens ${ }^{87}$ - advierten que la identidad de cada persona es producto de un contexto sociopolítico específico, definido por un conjunto de normas que afectan directamente en la conformación individual y colectiva de la identidad ${ }^{88}$. Las normas sociales, resultado de creencias y convenciones, constituyen el cuerpo moral que ordena el comportamiento, y que, en consecuencia, establece lo adecuado dentro de la comunidad. La modificación de las creencias o de las normas sociales constituiría, entonces, un cambio en la identidad. Más no se trata de principios sencillos de transformar. Su cambio requiere, en último término, de negociación. Ello no supone, empero, que los individuos actúen solo dentro del sistema. Cada persona posee niveles de movilidad entre el discurso colectivo de la identidad y sus experiencias personales que la llevan a reaccionar, bien aprobándolo, bien rechazándolo. Así, las respuestas a los discursos de la identidad serán tan numerosas como las personas que compongan la sociedad, cuyas experiencias individuales conllevarán, a su vez, múltiples discursos identitarios formulados desde la subjetividad ${ }^{89}$.

\footnotetext{
85 Gruen, Erich. 2010. Rethinking the Other in Antiquity, Princeton, Princeton University Press, 2010, pp. 1-5.

${ }^{86}$ Véase Revell, Louise. 2016. Ways of Being Roman, p. 8; Kolonsy-Ostrow, Ana y Lyons, Claire (eds.). 1997. Naked Trust: women, sexuality and gender in classical art and archaeology, Routledge, Londres, passim.

87 Las propuestas de Giddens, que advierten la relación entre los sujetos y las estructuras sociales en un ordenamiento de tipo dialéctico, suponen que las acciones de los hombres generan estructuras sociales cuyo rol es el establecimiento de normativas para controlar las acciones. En este sentido, el sistema de Giddens adquiere una condición de flexibilidad y de movilidad de las estructuras, cuya relación acción-estructuración conlleva cambios en la sociedad que afectan en el modo en que estas se definen a sí mismas. Véase Giddens, Anthony. 1995. La constitución de la sociedad: bases para la teoría de la estructuración, Amorrortu, Buenos Aires, pp. 14 y ss.

88 Said, Edward, "Cultura, identidad, historia", en: Schröeder, Gerhart y Breunincher, Helga. 2009. Teoría de la cultura, Argentina, Fondo de Cultura Económica, p. 39.

${ }^{89}$ Véase Revell, Louise. 2016. Ways of Being Roman, p. 10.
} 
Se trata de una perspectiva que plantea la identificación de un individuo hacia su grupo como resultado de un proceso de vinculación entre discursos propios y colectivos. En cuanto persona de experiencias conscientes, el individuo dialoga con las normas sociales, cuya adquisición supone la adecuación entre su subjetividad y los roles colectivos establecidos y esperados. En palabras de Revell: "nuestras acciones se ajustan a la idea de lo que es apropiado a aspectos específicos de nuestra identidad, como nuestro rango social o nuestra identidad étnica, y esta idea de apropiación se extrae de las normas sociales esperadas"90. Serían estas normas, en definitiva, las que definirían el modo en que los individuos conciben la colectividad como un todo, que, aun con diferencias, esto es, la definición del yo, se reconoce como cuerpo social. Del mismo modo, el desconocimeinto, rechazo o, en último término, contraste de las reglas de una comunidad con otra, conllevarían su proyección en discursos de oposición.

Estamos ante una teoría compleja, derivada de la etnicidad, que entiende la romanidad en términos plurales. Vinculando prácticas culturales y estructuras sociopolíticas, afirma que la identidad es resultado de un proceso mixto -individual y colectivo- que, sin adquirir formas homogéneas, de igual modo cohesiona.

A este respecto, resultan sumamente interesantes los planteamientos de la identidad múltiple, cuyo reconocimiento en el Imperio constituye uno de lo principales aportes de la investigación reciente. Las diversas romanidades se asocian a formas de expresión local, producto natural de la interacción local con el poder central. Las diferencias, pues, no se advierten necesariamente como discrepancias, sino como manifestaciones alternas de romanidad en un imperio de carácter poliétnico ${ }^{91}$.

Bajo estas consideraciones, la vinculación de un individuo con su comunidad local no constituiría una negación al poder central, toda vez que, como sujeto de múltiples roles, contaría con diversas vías para identificarse con la sociedad en diferentes grados. En esta línea, Witcher ${ }^{92}$, siguiendo a David ${ }^{93}$ y a Keaveney ${ }^{94}$, propone el análisis de la romanidad a partir de un modelo de escalas, que aluden, de manera complementaria, a tres niveles de identidad. En el primero, una romanidad global o general, que reconocen analógicamente como una suerte de identidad nacional. En el segundo, en una escala intermedia, una identidad de tipo regional. En el tercero, finalmente, una identificación local. Cada una, con sus características específicas, conciliarían y conformarían la romanidad.

\footnotetext{
90 Idem.

${ }^{91}$ Gruen, Erich. 1994. Cultural and National Identity in Republican Rome, Ithaca-New York, Cornell University Press, pp. 12 y ss; Revell, Louise. 2016. Ways of Being Roman, pp. 48-55.

92 Witcher, R. 2000. "Globalization and Roman Imperialism", pp. 213-225.

93 David, Jean-Michel. 1996. The Roman Conquest of Italy, Oxford, Blackwell, pp. 135-139.

${ }^{94}$ Keaveney, Arthur. 1987. Rome and the Unification of Italy, Londres-Sidney, Croom Helm, p. 35.
} 
El análisis de Witcher, aplicado a Italia, evidencia diferentes respuestas locales ante el avance militar romano, proceso en que las identidades étnicas y culturales se crearon y destruyeron, se fortalecieron y debilitaron, se purificaron y mezclaron, hasta finalmente decantar en una nueva conformada en la confluencia de ambas culturas ${ }^{95}$. Así lo advierte Roselaar $^{96}$ al señalar la inclusión de las etnias occidentales en un proceso de integración organizado, cuyo propósito se habría dirigido a la conservación de aspectos locales. Precisamente por ello apunta la integración como un "proceso cultural por el cual dos o más pueblos diferentes vienen a compartir la misma cultura e identidad" ${ }^{\prime 7}$, lo que supone cambios en función de la adaptación a estilos de vida, hábitos y costumbres diferentes. En definitiva, un análisis que advierte un proceso de construcción identitaria de carácter paradójico, que concilió la similitud y la diferencia mediante estrategias no solo destinadas para ello, sino surgidas o adaptadas en función de cada localidad ${ }^{98}$; que conllevaron, a su vez, diversas formas de romanidad en el escenario multicultural del Mediterráneo ${ }^{99}$.

Habida cuenta del extenso escenario en que Roma desplegó su expansión, la identidad cultural ha intentado referir los factores que influyeron en la construcción de la romanidad, lo que ha supuesto retomar temas, problemas y términos profundamente criticados por los estudios postcoloniales. Imperialismo y romanización constituyen buen ejemplo. A la luz de una nueva mirada -que, por cierto, resulta menos ideologizada-, han adquirido nuevo sentido, donde la ciudad encuentra especial reconocimiento. Tanto el análisis de la cultura material como de las fuentes literarias establecen en esta un rol identitario de primer orden, lo que se advierte en función de su carácter simbólico y social.

En cuanto a la organización física, la ciudad constituía un modelo de ordenamiento homólogo de lo que era Roma en las provincias, posibilitando establecer analogías entre estas. La arquitectura, el tipo de edificaciones y el uso del espacio no solo constituían un modelo de organización urbana, sino que representarían, mediante íconos simbólicos, las formas de vida romanas susceptibles de conocer y asumir en las actividades de la vida cotidiana ${ }^{100}$. Las edificaciones situadas en el foro ofrecían la imagen que la Roma misma pretendía mostrar de sí, erigiéndose como instrumentos para irradiar la cultura en el funcionamiento cotidiano de las

\footnotetext{
95 Witcher, R. 2000. “Globalization and Roman Imperialism, p. 224.

${ }^{96}$ Roselaar, Saskia. 2015. "Introduction”, en Roselaar, Saskia (ed.). Process of Cultural Change, pp. 1-2.

97 Ibid., p. 3.

98 Revell, Louise. 2009. Roman Imperialism and Local Identities, New York, Cambridge University Press, p. 140.

99 Véase Gruen, Erich. 1993. "Cultural Fictions and Cultural Identity", en Transactions of the American Philological Asociation, Vol. 123, 1993, pp. 1-4.

100 En esta línea encontramos a Lomas, Kathryn. 1998. "Roman Imperialism and the city in Italy", en Laurence, Ray y Berry, Joanne (eds.). Cultural Identity in the Roman Empire, Londres-Nueva York, Routledge, pp. 64-76; Revell, Louise. 2009. Roman Imperialism, pp. 1-40; Fornis, César. 2007. "La construcción de la identidad romana en Corintio", en Habis, №38, pp. 213-215.
} 
instituciones. Asimismo, actividades comunes como la asistencia a los baños públicos o a los espectáculos de gladiadores permitían establecer comunicación entre los habitantes de las provincias, que comprendían y actuaban en estos desde su propia interpretación del ser romano. Se trataría de un proceso de apropiación de la identidad romana, expresada desde diversas formas de comprenderse y vivirse. Así, "un grupo de personas, en un evento público, podía expresar su romanidad común y al mismo tiempo expresar su rango social a través de sus roles específicos" ${ }^{101}$.

Por su parte, el ejercicio del poder político y jurídico también sería un recurso a la construcción de la identidad. Mediante el desempeño de magistraturas y de otros cargos administrativos se intentaría exhibir modelos de comportamiento romano, que a poco andar se transformarían en ideales asumidos y adecuados por los sujetos vinculados a la administración romana ${ }^{102}$. Con todo, se trataría de estructuras lo suficientemente flexibles, cuyo contenido, modelado de manera bilateral, conllevó múltiples formas de comprender la romanidad. De ahí que la ciudad se inscribiera, a la vez que como instrumento de exhibición de la romanidad, como "elemento autoidentificatorio de los indígenas como individuos" 103.

En definitiva, el desarrollo de la identidad cultural ha permitido construir una visión de la identidad romana desde miradas amplias, centradas en las características multiculturales y poliétnicas de Roma. No obstante, aun cuando constituyen un aporte, no dejan de presentarnos ciertas dudas. Así, por ejemplo, la relevancia que otorgan a factores como el género y la edad resultan sumamente complejos de identificar y precisar, puesto que, aunque son categorías específicas, son, a su vez, de carácter universal, transversales a todos los estratos sociales. Asimismo, la importancia otorgada a estos aspectos pareciera exagerar niveles de consciencia en grupos de difícil acceso, como niños, mujeres y esclavos.

Por su parte, las teorías de género, vinculadas a las corrientes feministas, nos parecen inabordables en la Antigüedad. Resulta anacrónico identificar estas categorías en el mundo clásico, desarrollado desde códigos sociales y culturales propios. Comprendemos el esfuerzo por entender la historia romana en términos comparados; sin embargo, este tipo de interpretaciones, desde categorías modernas, adaptan criterios de análisis a escenarios temporal y paradigmáticamente disímiles. Del mismo modo, el exceso de variables ha quitado contenido al significado de la identidad. Si consideramos que esta se encuentra en todo lo

\footnotetext{
${ }^{101}$ Revell, Louise. 2009. Roman Imperialism, p. 193.

102 Braund, David. 2001. "Cohors: the governor and his encourage in the self-image of the Roman Republic", en Laurence, Ray y Berry, Joanne (eds.). Cultural Identity in the Roman Empire, pp. 10-23; Laurence, Ray. 2001. "Introduction", en Laurence, Ray y Berry, Joanne (eds.). Cultural Identity in the Roman Empire, pp. 2-3.

103 Pina Polo, Francisco. 2011. "Etnia, ciudad y provincia en la hispana republicana”, en Caballos, Antonio y Lefebvre, Sabine (comp.), Roma generadora de identidades, Madrid, Casa de Velázquez, p. 51.
} 
referente al ser humano, resulta, sin duda, inabordable. Es, por ello, necesario establecer parámetros para comprender, más que la romanidad misma, sus formas definitorias.

Finalmente, nos parece que si bien los estudios centrados en detalles y múltiples roles sociales son interesantes, han apartado el principal problema en cuestión, esto es, qué es ser romano, o, en último término, qué significaba serlo para Roma. No podemos comprender la identidad sin un elemento unificador, sin un aspecto común. Nos parece imposible hablar de una comunidad romana sin concebir que algo la comunicara y definiera, lo que supone omitir, al menos parcialmente, sus discrepancias.

\section{De la identidad cultural a la identidad ciudadana}

La relevancia de las ciudades en la construcción de la identidad, promovida inicialmente por los estudios de enfoque cultural, ha retomado la vía de análisis centrada en el vínculo más relevante entre los individuos y Roma como entidad sociopolitica y cultural, a saber, la civitas. En cuanto estatuto ciudadano, la civitas permite aludir una relación de carácter global entre las provincias y el poder central en una perspectiva que no disputa con los rasgos específicos de cada comunidad, puesto que, respetando sus particularidades, constituye un vínculo o reconocimiento entre Roma y sus miembros más allá de sus diferencias étnicas, geográficas, lingüísticas o históricas ${ }^{104}$.

Si bien cada ciudad constituyó una realidad en sí misma, con una identidad ligada a prácticas socioculturales y a normas de comportamiento regional, el ámbito ciudadano, ordenado por el derecho romano, construyó una comunidad universal, ofreciendo categorías de pertenencia en términos jurídicos y culturales. En efecto, las clasificaciones de la ley romana, específicas en cuanto a derechos y deberes, establecían un conjunto de características que definían a los ciudadanos romanos de pleno derecho, lo que no solo aludía a aspectos de carácter legal, sino también cultural. Ser ciudadano romano suponía formar parte de una comunidad definida y delimitada por un conjunto de normas, cuya aplicación en el ámbito político conllevaba un modelo de organización social vinculado, además, a un código de comportamiento específico en cuanto a las prácticas públicas permitidas y prohibidas. De este modo, la ciudadanía fue más que una fórmula jurídica y administrativa. Fue el reconocimiento como miembro por parte de la colectividad romana misma ${ }^{105}$.

\footnotetext{
${ }^{104}$ Véase Gardner, Jane. 1993. Being a Roman Citizen, Routledge, London-New York, pp. 1 y ss; Andrés, Francisco. 2007. “Ciudadanía romana y cosmopolitismo moderno", en HAnt, № XXXI, pp. 257-259.

105 Heater, Derek. 2007. Ciudadanía. Una breve historia, Madrid, Alianza, p. 63; Horrach, Juan. 2009. "Sobre el concepto de ciudadanía: historia y modelos", en Factórum, №6, pp. 7-8; Blanch, José. 2013. "Dignidad personal y libertad: libertad y ciudadanía en la antigua Roma", en Anuario de la Facultad de Derecho de la Universidad Autónima de Madrid, № 17, p. 167-168.
} 
Desde esta perspectiva, David afirma que ser romano conllevaría la aceptación global de las lógicas de una estructura sociopolítica que configuraron la identidad colectiva, y que, por lo mismo, supuso un estatus definido, normado y delimitado en su "acceso" ${ }^{106}$. La ciudadanía romana indicaría, por un lado, la pertenencia a un cuerpo social delimitado bajo normas de comportamiento esperado, $\mathrm{y}$, por otro, un estatus referido por ese reconocimiento de miembro, que supondría -antes de su extensión generalizada durante el Imperio- una condición de privilegio ligada a derechos políticos y económicos. Así entendida, la civitas constituiría el sello de pertenencia a Roma, reconocible por romanos y por quienes no lo eran.

Los análisis de la identidad vinculada a la ciudadanía habían sido referidos por estudiosos relacionados con las visiones más clásicas de la historia romana, ligados, incluso, al paradigma de la romanización. La civitas constituía un tópico relevante para señalar vínculos jurídicosociales, y se entendía como el aspecto más relevante de la romanidad. La ciudad, entendida como una realidad política y cultural romana desenvuelta temporal y espacialmente, se asociaba con una carga simbólica de romanidad, conformada por aspectos como el pasado, la institucionalidad, la religiosidad $y$, en suma, todo elemento inherente a la cultura, que traspasado en la constitución del ámbito cívico habrían devenido en una mentalidad específica ${ }^{107}$.

Ahora bien, a diferencia de los estudios de la romanización, la ciudadanía-identidad en términos culturales centra su atención en la capacidad de la civitas para representar lo romano en diferentes escenarios. Bajo esta perspectiva, todo el contenido definitorio de la civitas se establecería como criterio para construir una idea de romanidad, que se haría explícita en las representaciones sociales manifiestas en los productos de la cultura. Por ello, cultura material, representaciones literarias y artísticas y, en fin, toda expresión de la sociedad, posibilitaría aludir el ser romano, definido a través de los límites demarcados por la ciudadanía. Así, las definiciones de la romanidad desde la ciudadanía no solo permitirían referir características del romano, sino de aquellos que paulatinamente se vincularon a la ciudad, que a la postre obtendrían la ciudadanía producto de una transformación.

A este respecto, Roselaar advierte que el contacto entre las culturas romana e itálicas modificaron el modo en que los individuos se veían a sí mismos, situación en que la ciudadanía, en tanto definición cívica, posibilitaba la confluencia de ambas comunidades bajo un horizonte

\footnotetext{
106 David, Jean-Michel. 2000. La République romaine de la deuxième guerre punique à la bataille d'Actium 281-31, París, Editions du Seuil, pp. 19-20.

107 En esta línea encontramos a Finley, Moses. 1973. The Ancient Economy, California, niversity of California Press, p. 47; Sherwin-White, Adrian. 1973. The Roman Citizenship, pp. 402 y ss; Grimal, Pierre. 1991. Las ciudades romanas, Oikos-Tau, Barcelona, p. 6; Hubeñák, Florencio. 1987. "Terra et urbs: la búsqueda de la mentalidad del ciudadano de la Roma Republicana", en Res Gesta, №22, p. 139; Harris, William. 2002. "Roma vista desde afuera", pp. 52; Bancalari, Alejandro. 2015. La idea de Europa en el mundo romano, Chile, Editorial Universitaria, pp. 85-90; Herrera, Héctor. 1990-1991. "La constitución del ámbito cívico en el mundo grecorromano", en Limes, № 2, pp. 21-24, 36.
} 
común. Otorgando comprensión y sentido a la construcción de una nueva cultura, ofrecía criterios para delimitar las "ideas que tenían las partes involucradas sobre su lugar en el mundo y su relación con las demás" ${ }^{108}$. Se trata de una perspectiva que pretende comprender la forma en que los romanos se veían a sí mismos frente al orbe y en la historia, cuyo principio referencial sería la condición ciudadana. El estatuto jurídico supondría el reconocimiento bajo el cuerpo legal de la ciudad y del estado; la carga valórica, el de orden identitario.

Bajo estas consideraciones, la relevancia de la ciudadanía como agente de la romanidad se comprende por el carácter simbólico del concepto y, asimismo, por la connotación comunitaria de su constitución y potestad. Como advierten Caballos y Lefebvre ${ }^{109}$, el imperium romano no se ejercía sobre territorios geográficos o sobre grupos de individuos cualesquiera, sino ante comunidades políticas que, vinculadas, conformaban el escenario cívico romano, definido y comprendido desde su lugar en el orbis romanus. De ahí que las delimitaciones de la civitas constituyeran un criterio para señalar la romanidad desde circunstancias y lugares heterogéneos.

Nos encontramos ante una concepción de la ciudadanía en razón de su funcionalidad, que habría permitido aunar comunidades étnicamente dispares mediante la construcción de un sistema de representación. Así lo advierte Emma Dench $^{110}$ en un importante estudio de los pueblos de los Apeninos centrales, identificando en la ciudadanía una amplia capacidad de adaptación y referencia. Adaptación, por cuanto admite su extensión a miembros de variada procedencia; y referencia, toda vez que se transformaba en un criterio de representación de la romanidad. Desde las principales fuentes literarias reconoce la mirada cambiante de Roma ante los pueblos Apeninos en virtud de su contacto e incorporación a la ciudad, advirtiendo un vuelco total entre la barbarie atribuida previo contacto con los sistemas cívicos romanos y su posterior representación como civilizados, asumidos como miembros de la ciudad. Como referente de la identidad romana, la civitas sería un concepto adaptable a la realidad multiétnica de Roma, susceptible de referir como conjunto, precisamente, por su connotación sociocultural, más allá de su sentir jurídico ${ }^{111}$.

Con todo, el carácter extensivo de la civitas no se aleja del marco institucional de la urbe. Al menos así lo vemos desde los análisis de Arno, quien, a través de las principales fuentes

\footnotetext{
108 Roselaar, Saskia. 2012. "Introduction”, en Roselaar, Saskia (ed.). Process of Integration and Identity Formation in the Roman Republic, Leiden-Boston, Brill, p. 9; Roselaar, Saskia (ed.). 2015. Process of Cultural Change, pp. 9-10.

109 Caballos, Antonio y Lefebvre, Sabine. 2011. "Introducción”, en Caballos, Antonio y Lefebvre, Sabine (comp.), Roma generadora de identidades, p. 2.

110 Dench, Emma. 1995. From Barbarians to New Men. Greek, Roman, and Modern Perceptions of Peoples of the Central Apennines, New York, Clarendon Press, pp. 10 y ss.

${ }^{111}$ Dench, Emma. 2005. Romulus' Asylum. Roman Identities from the Age of Alexander to the Age of Hadrian, New York, Oxford University Press, pp. 3-4.
} 
literarias y materiales de la Tardorrepública, advierte en la ciudadanía un recurso para construir una comunidad de amplio alcance y para expresar la ideología imperial. Asumiendo la comunidad como la "colección de individuos cuyos miembros se ven a sí mismos y son vistos como seres distintos de cualquier otra colección de individuos" ${ }^{\prime 12}$, entiende que el orden institucional romano, simbolizado por la ciudadanía, posibilitaría la conformación de colectividades con identidad supraestatal ${ }^{113}$, ligadas al marco de comportamiento de la romanidad. De modo que la adhesión a la romanidad sería, bajo el principio de la ciudadanía, una capacidad para ser representado desde sus cánones y reconocido como parte de una institucionalidad común. En definitiva, una alusión a la ciudadanía a partir de sus posibilidades de adaptación a diferentes escenarios y pueblos, cuyo contenido se estableció como el eje de la construcción identitaria en las provincias mediante la "readaptación de las identidades y pertenencias previas, de sus manifestaciones sociales y vivencias internas"114.

Es precisamente en esta misma línea que lo plantea Stee ${ }^{115}$ para el período Republicano, quien, a partir de Cicerón, alude al modo en que el acceso a la ciudadanía supuso un canon estable para referir la romanidad. De acuerdo con Steel, las solicitudes de ciudadanía y los casos de repetundae tratados por Cicerón promueven una imagen de la romanidad en función de la civitas, cuya construcción conceptual ligada a la justicia y al marco valórico de la cultura constituyeron el fundamento de la correcta práctica política y social de un romano.

Aun cuando la ciudadanía como criterio y objeto de la identidad no se restringe, en principio, a las fuentes literarias, su estudio ha sido resultado efectivo de un retorno a los textos. No se trata, sin embargo, de un análisis al estilo clásico, sino desde perspectivas novedosas, centradas incluso en géneros literarios antes omitidos para este tipo de aproximaciones ${ }^{116}$. Ahora bien, aun cuando las definiciones ciudadanas constituyen una vía de análisis interesante, las diferencias entre quienes proponen sus fundamentos legales y quienes advierten una mirada más bien cultural -ligada a sus capacidades de representación- evidencian la complejidad de establecer un criterio unívoco a toda la historia de Roma. Desde la dimensión jurídica o la cultural, la ciudadanía, como alusivo a la romanidad, nos parece un referente todavía general de la misma; tratándose de un concepto que, además, no puede disociarse. Ciudadanía es sin duda un referente cultural, pero fundamentado en principios jurídicos. Desde esta perspectiva,

\footnotetext{
112 Arno, Claudia. 2012. How Romans Become Roman, pp. 12-13.

113 íbid., p. 208.

114 Wulff, Fernando. "Hablando de identidades. Reflexiones historiográficas sobre Italia entre la República y el Imperio", en Caballos, Antonio y Lefebvre, Sabine (comp.), Roma generadora de identidades, p. 34.

115 Steel, C., Cicero, Rhetoric, and Empire, Oxford University Press, New York, 2001, pp. 8-10; 73-76.

${ }^{116}$ Véase Gunderson, Erik. 2003. Declamation, Paternity and Roman Identity. Authority and the Rethorical Self, New York, Cambridge University Press, pp. 22-24.
} 
si bien el concepto de identidad ciudadana constituye una fórmula legítima para referir algunos periodos del devenir romano, para otros resulta sumamente complejo.

La Tardorrepública, en tanto proceso de crisis política y social fue, por sobre todo, una crisis jurídica, en la que el concepto de ciudadanía fue profundamente afectado; la referencia a la identidad como ciudadanía dejó de tener sentido, y aparecieron, por ello, otros elementos vinculados a la romanitas. En este sentido, la civitas representa un punto de partida para comprender las características de la identidad romana bajo algunas circunstancias, pero también un objeto de análisis para referir su quiebre. Con todo, la perspectiva de la civitas ha devuelto el protagonismo al estudio de la identidad romana propiamente tal, permitiendo retomar una vía obscurecida por los estudios de la etnicidad y por las posturas ideológicas; ofreciendo, además, un espacio para el desarrollo de visiones complementarias. Asimismo, la referencia a la ciudadanía ha dirigido los estudios de la identidad en una vía definida, solventando en parte la heterogeneidad propuesta por los análisis de la identidad cultural, aun cuando ha surgido, al menos en principio, desde esta.

\section{Conclusiones}

El recorrido de los estudios de la identidad romana desde fines del siglo XIX hasta nuestros días muestra el diálogo entre los problemas contextuales, historiográficos y conceptuales de la romanidad. Desde miradas principalmente políticas y homogeneizantes centradas en la romanización clásica, los estudios de la etnicidad han propiciado una nueva aproximación, así como también nuevas vías y agentes constructores de la identidad en las provincias. Son estudios del todo sugerentes, pero también cargados de una ideología reivindicativa y nativista que nubla y en ocasiones fuerza la interpretación de las fuentes arqueológicas.

El surgimiento de los paradigmas culturales, por su parte, ha generado el diálogo entre ambas corrientes, propiciando una nueva lectura de las categorías y fundamentos tradicionales de la romanidad. Precisamente desde esta línea, los análisis a la identidad se han abocado al estudio de la civitas, cuya fuerza convergente permite comprender las características de una diversidad unificada en términos jurídicos y valóricos característicos de la comunidad multiétnica romana. Se trata de un concepto fundamental a la historia romana, toda vez que se presenta transversalmente en su historia y adquirió matices diversos en función del contexto sociocultural del período monárquico, republicano e imperial.

El problema con la ciudadanía, empero, es que resulta inescindible de su carácter jurídico, cuya fuerza y coherencia sufrió notables quiebres en períodos de crisis de la historia de la Urbs. Nos parece, por tanto, que es un concepto relevante, pero no del todo definitorio de la identidad, que, por su multidimensionalidad, probablemente adquirió múltiples formas de vinculación y de definición. La síntesis que ofrecimos muestra, de este modo, la heterogeneidad 
de un concepto que, fruto de múltiples interpretaciones, evidencia la necesidad de profundizarse en función de los períodos particulares de la historia romana.

\section{Bibliografía}

Adams, J. 2003. Bilingualism and the Latin Language, Cambridge, Cambridge University Press.

Alcock, S. 1997. "The problem of romanization, the power of Athens", en Hoff, M. y Rotroff, S. (eds.). The Romanization of Athens, Oxford, Oxbow.

Alonso-Nuñez, J. 1989. "Reflexiones sobre el imperialismo romano en Hispania”, en Studia Histórica, Historia Antigua, №7, España, pp. 7-10.

Andrés, F. 2007. “Ciudadanía romana y cosmopolitismo moderno", en Hispania Antqua, № XXXI, España, pp. 253-266.

Arno, C. 2012. How Romans Became "Roman": Creating Identities in an Expanding World, Michigan, Universidad de Michigan, Tesis Doctoral.

Bancalari, A. 2005. "Theodor Mommsen, el mundo romano y sus proyecciones: a propósito del centenario de su muerte", en Atenea № 492, Concepción, pp. 135-146.

Bancalari, A. 2007. Orbe romano e imperio global. La romanización desde Augusto a Caracalla, Santiago, Editorial Universitaria.

Bancalari, A. 2015. La idea de Europa en el mundo romano, Chile, Editorial Universitaria.

Barrett, J. 1997. "Romanization: a critical comment", en Mattingly, D. (ed.). Dialogues in Roman Imperialism, JRASS, №23, Rode Island, Portsmouth.

Bartel, B., 1980. "Colonialism and cultural responses: problems related to Roman provincial analysis", en World Archaeology №12, pp. 11-26.

Barth, F. 1969. "Introduction”, en Barth, F. (ed.). Ethnic Groups and Boundaries, Boston, Little Brown.

Bartlett, R. 1994. The Making of Modern Europe. Conquest, Colonization and Cultural Change, Inglaterra, Penguin.

Bauman, R. A. 2000. Human Rights in Ancient Rome, Londres-Nueva York, Routledge.

Beltrán, F. 1999. "Writing, language and society: Iberians, Celts and Romans in Northeastern Spain in the 2nd and 1st centuries B. C.", en Bulletin of the Institute of Classical Studies, № 43, pp. 131-151.

Beltrán, F. 2011. “Lengua e identidad en la Hispania Romana”, en Paleohispánica, №11, pp. 19-59. Bénabou, M. 1976. La résistance africane à la romanization, París, Éditions la Découverte.

Bénabou, M. 1978. “Les Romains ont-ils conquis l'Afrique?”, en Annales: Économies, Sociétés, Civilisation, año 33, № 1, pp. 83-88.

Blanch, J. 2013. “Dignidad personal y libertad: libertad y ciudadanía en la antigua Roma”, en Anuario de la Facultad de Derecho de la Universidad Autónima de Madrid, № 17, pp. 163-182.

Braund, D. 2001. "Cohors: the governor and his encourage in the self-image of the Roman Republic", en Laurence, R. y Berry, J. (eds.). Cultural Identity in the Roman Empire, Londres-Nueva York, Routledge.

Brunt, P. A. 1976. "The Romanization of local ruling classes in the roman empire", en Pippidi, D. M. (ed.), Assimilation et résistance à la culture grecorromaine dans le monde ancien, Paris, Les Belles Lettres.

Buono-Core, R. 2006. “Un breve análisis de la historiografía griega sobre la antigua Grecia en los siglos XIX y XX”, en Europa №4, Mendoza, pp. 5-72.

Caballos, A. y Lefebvre, S. (comp.). 2011. Roma generadora de identidades, Madrid, Casa de Velázquez. Cohen, A. 1974. Urban Ethnicity, Londres, Tavistock Publications.

Comaroff, John y Comaroff, Jean. 2011. Etnicidad S.A., Madrid, Katz. 
Crawley, J. 2003. "Roman Africa", en Merryweather, A. y Prag, J. (eds.). Digressses, Londres, University of London.

David, J-M. 1996. The Roman Conquest of Italy, Oxford, Blackwell.

David, J-M. 2000. La République romaine de la deuxième guerre punique à la bataille d'Actium 281-31, París, Editions du Seuil.

Dench, E. 1995. From Barbarians to New Men. Greek, Roman, and Modern Perceptions of Peoples of the Central Apennines, New York, Clarendon Press.

Dench, E. 2005. Romulus' Asylum. Roman Identities from the Age of Alexander to the Age of Hadrian, New York, Oxford University Press.

Desideri, P. 1991. “La romanizzazione dell 'impero”, en Schiavone, A. (ed.), Storia di Roma, Vol. 2, Torino, Einaudi.

Fear, A. 1996. Rome and Baetica: Urbanizarion in Southern Spain c.50 BC-AD 150, Oxford, Clarendon Press.

Finley, M. 1973. The Ancient Economy, California, University of California Press.

Fornis, C. 2007. "La construcción de la identidad romana en Corintio", en Habis №38, pp. 205-224.

Freeman, P. 1997. "Mommsen to Haverfield: the origins of studies of romanization”, en Mattingly, D. (ed.). Dialogues in Roman Imperialism, JRASS, №23, Rode Island, Portsmouth.

Gabba, E. 2000. "El problema de la "unidad" de la Italia romana", en Gabba, E. y Laffi, U., Sociedad y política en la Roma Republicana, Pisa, Pacini.

Gardner, A. 2013. "Thinking about Roman imperialism: postcolonialism, globalization and beyond?", en Britannia №44, pp. 1-25.

Gardner, J. 1993. Being a Roman Citizen, London-New York, Routledge.

Geertz, C. 1963. "The integrative revolution: primordial sentiments and civil politics in the new states", en Geertz, C. (ed.). Old Societies and New States, New York, The Free Press.

Giddens, A. 1995. La constitución de la sociedad: bases para la teoría de la estructuración, Buenos Aires, Amorrortu.

Grimal, P. 1991. Las ciudades romanas, Barcelona, Oikos-Tau.

Gruen, E. 1993. "Cultural Fictions and Cultural Identity", en Transactions of the American Philological Asociation, Vol. 123, pp. 1-14.

Gruen, E. 1994. Cultural and National Identity in Republican Rome, Ithaca-New York, Cornell University Press.

Gruen, E. 2010. Rethinking the Other in Antiquity, Princeton, Princeton University Press.

Gunderson, E. 2003. Declamation, Paternity and Roman Identity. Authority and the Rethorical Self, New York, Cambridge University Press.

Habinek, T. 2001. The Politics of Latin Literature, Princeton, Princeton University Press. Harris, W. 1979. War and Imperialism in Republican Rome, USA, Oxford Universisty Press. Harris, W. 2002. "Roma vista desde afuera", en Semana de estudios romanos, Vol. XI. Haselgrove, C. 1984. "Romanization before conquest: Gaulish precedent and British consequences", en Blagg, T. y King, A. (eds.). Military and Civilian in Roman Britain. Cultural relationships in a frontier province, Vol. 136, Oxford, BAR British Academy.

Haverfield, F. 1915. The Romanization of Roman Britain, London, Oxford University Press. Heater, D. 2007. Ciudadanía. Una breve historia, Madrid, Alianza.

Herrera, H. 1990-1991. “La constitución del ámbito cívico en el mundo grecorromano”, en Limes № 2, Santiago, pp. 403-429.

Hingley, R. 1997. "Resistance and domination: social change in Roman Britain”, en Mattingly, D. (ed.). Dialogues in Roman Imperialism, JRASS, №23, Rode Island, Portsmouth. 
Hingley, R. 2000. Roman Officers and English Gentlemen. The Imperial Origins of Roman Archaeology, Routledge, London-New York.

Hingley, R. 2005. Globalizing Roman Culture: Unity, Diversity and Empire, Londres-New York, Rutledge. Hitcher, B. 2007. The First Globalization: The Roman Empire and Its Legacy in the 21st Century, Oxford, Oxford University Press.

Hobsbawm, E. 2004. Naciones y nacionalismo desde 1789, Barcelona, Crítica.

Horrach, J. 2009. "Sobre el concepto de ciudadanía: historia y modelos", en Factórum, №6, pp. 1-22.

Hubeñák, F. 1987. "Terra et urbs: la búsqueda de la mentalidad del ciudadano de la Roma

Republicana", en Res Gesta, №22.

Hurtado, M. 2005. Introducción a la historia antigua. Caracas, Fondo de Humanidades.

Hyun Jin, J. 2009. Ethnicity in Ancient Greece and China, Londres, Duckworth.

Isaacs, H. 1974. "Basic group identity: ideals of the tribe", en Ethnicity, Vol. 15, № 1.

Isaacs, H. 1974. "Basic group identity", p. 30; Keyes, Charles. 1976. "Towards a new formulation of the concepto of ethnic grup", en Ethnicity, №3.

James, S. 2001. "Romanization and the peoples of Britain", en Keay, S. y Terrenato, N. (eds.). Italy and the West: Comparative Issues in Romanization, Oxford, Oxbow.

Jones, M. y Miles, D. 1979. "Celts and Romans in the Thames Valley: approaches to culture change", en Burnham, B. y Johnson, H. (eds.). Invasion and Response: The case of Roman Britain, Oxford, BAR British Series, №3.

Jones, S. 1997. The Archaeology of Ethnicity, Londres-Nueva York, Routledge.

Keaveney, A. 1987. Rome and the Unification of Italy, Londres-Sidney, Croom Helm.

Keay, S. y Terrenato, N. (eds.). 2001. Italy and the West: Comparative Issues in Romanization, Oxford, Oxbow.

Kellas, J. 1991. The Politics of Nationalism and Ethnicity, Londres, Macmillan.

Kolonsy-Ostrow, A. y Lyons, C. (eds.). 1997. Naked Trust: women, sexuality and gender in classical art and archaeology, Routledge, Londres.

Laurence, R. y Berry, J. (eds.). 2001. Cultural Identity in the Roman Empire, Londres-Nueva York, Routledge.

Laurence, R. 2001. "Roman narratives: the writing of archaeological discourse - a view from Britain?", en Archaeological Dialogues, № 8, pp. 90-100.

Leveau, P. 1978. "La situación coloniale de'IAfrique romaine”, en Annales: Économies, Sociétés, Civilisation, año 33, №1, pp. 89-92.

Mattingly, D. (ed.). 1997. Dialogues in Roman Imperialism, JRASS, №23, Rode Island, Portsmouth.

Mattingly, D. 2002. "Vulgar and weak 'Romanization' or time for a paradigm shift", en Journal of Roman Archaeology, №15, pp. 536-540.

Mattingly, D. 2011. Imperialism, Power and Identity, Princeton-Oxford, Princeton University Press.

McKai, J. 1982. "An exploratory synthesis of primordial and mobilizationist approaches to ethnic phenomena", en Ethnic and Racial Studies, Vol. 5, pp. 401-402.

Millet, M. 1990. The Romanization of Britain: an essay in archaeological interpretation, Cambridge, Cambridge University Press.

Millet, M. 2003-2004. “The Romanization of Britain: changing perspectives", en Kodai: Journal of Ancient History, №13-14, pp. 169-173.

Mommsen, T. 1945. El mundo de los Césares, Fondo de Cultura Económica, México D. F.

Nash, M. 1989. The Cauldron of Ethnicity in the Modern World, Chicago, University of Chicago Press.

Pina Polo, F. 2011. "Etnia, ciudad y provincia en la hispana republicana", en Caballos, A. y Lefebvre, S.

(comp.), Roma generadora de identidades, Madrid, Casa de Velázquez.

Prosdocimi, A. (ed.). 1978. Popoli e civilità dell'Italia antica, Roma, Biblioteca di Storia Patria. 
Reece, R. 1980. "Town and country: the end of Roman Britain", en World Archaeology, Vol. 12, pp. 7792.

Revell, L. 2009. Roman Imperialism and Local Identities, New York, Cambridge University Press.

Revell, L. 2016. Ways of Being Roman, Oxford-Filadelfia, Oxbow Books.

Río, M. 2002. "Visiones de la etnicidad", en Revista española de investigaciones sociológicas, Vol. 98, pp. 85-96.

Roces, W. 1945. "Prólogo", en Mommsen, T. El Mundo de los Césares, México D. F., Fondo de Cultura Económica.

Roselaar, S. (ed.). 2015. Process of Cultural Change and Integration in the Roman World, LeidenLondres, Brill.

Roselaar, S. 2012. "Introduction", en Roselaar, S. (ed.). Process of Integration and Identity Formation in the Roman Republic, Leiden-Boston, Brill.

Said, E. 2009. "Cultura, identidad, historia”, en Schröeder, G. y Breunincher, H. Teoría de la cultura, Argentina, Fondo de Cultura Económica.

Sancho, L. (coord.). 2015. La Antigüedad como paradigma. Espejismos, mitos y silencios en el uso de la historia del mundo clásico por los modernos. Zaragoza, Universidad de Zaragoza.

Scopacasa, R. 2015. "An Alllied of Integration: Italian Elites and Consumption in the Second Century B. C.", en Roselaar, S. (ed.). Process of Cultural Change and Integration in the Roman World, LeidenLondres, Brill.

Sherwin-White, A. 1973. The Roman Citizenship, Oxford, Oxford University Press.

Shils, E. 1957. Center and Perisphery: essays in macrosociology. Selected papers of Edward Shils, Vol. II, Chicago, Chicago University Press.

Smith, A. 1986. The Ethnic Origins of Nations, Oxford, Blackwells.

Smith, J. 1997. Roman Villas: A study in Social Structure, Londres, Routledge.

Steel, C. 2001. Cicero, Rhetoric, and Empire, New York, Oxford University Press.

Sweetman, R. 2007. "Roman Knossos: The Nature of a Globalized City", en American Journal of

Archaeology, Vol. 111, №1, pp. 61-81.

Terrenato, N. 1998. "The Romanization of Italy: Global Acculturation or Cultural Bricolage?", en Forced, C., Hawthorne, J. y Witcher, R. (eds.), Theoretical Roman Archaeology Journal, № 97.

Torelli, M. 1995. Studies in the romanization of Italy, Canadá, University of Alberta Press.

Twedie, F. 2015. "Volterrae and the Gens Caesina", en Roselaar, S. (ed.). Process of Cultural Change and Integration in the Roman World, Leiden-Londres, Brill.

Wallace-Hadrill, A. 2000. "The roman revolution and material culture", en Paschoud, F., Giovanni, A. y Grange, B. (eds.). La Revolution Romaine après Ronald Syme: Bilans at perspectives, Entretiens sur I'antiquité classique, Geneva, Vandoeuvres.

Weber, M. 1978. Economy and Society. An Outline of Interpretative Sociology, Berkeley-Los AngelesLondres, University of California Press.

Webster, J. 2001. "Creolizing the Roman Provinces", en American Journal of Archaeology, № 105, pp. 209-225.

Wells, P. 2004. "The Barbarians Speak: How the Conquered Peoples Shaped Roman Empire”, en Champion, C. (ed.). Roman Imperialism: Readings and Sources, Estados Unidos, Blackwell Publishing. Witcher, R. 2000. "Globalization and Roman Imperialism: perspectives on identities in Roman Italy", en Herring, E. y Lomas, K. (eds.). The Emergence of State Identities in Italy in the First Millenium BC., Londres, Accordia Research Institute.

Woolf, G. 1997. "Beyond Roman and Natives", en World Archaeology, Vol. 28, №3, pp. 339-350. Woolf, G. 1998. Becoming Roman. The origins of provincial civilization in Gaul, Cambridge, Cambridge University Press. 
Woolf, G. 2001. "The Roman cultural revolution in Gaul”, en Keay, S. y Terrenato, N. (eds.). Italy and the West: Comparative Issues in Romanization, Oxford, Oxbow.

Wulff, F. "Hablando de identidades. Reflexiones historiográficas sobre Italia entre la República y el Imperio", en Caballos, A. y Lefebvre, S. (comp.), Roma generadora de identidades, Madrid, Casa de Velázquez.

Wulff, F. 2007. "Las provincias de Hispania en época Republicana: una reflexión sobre enfoques y perspectivas", en Santos, J. y Torregaray, E. (eds.). Laudes provinciarum: retórica y política en la representación del imperio romano, Vitoria, Universidad del País Vasco. 